\title{
Different effects of constitutive and induced microbiota modulation on microglia in a mouse model of Alzheimer's disease
}

Charlotte Mezö ${ }^{1,2}$, Nikolaos Dokalis ${ }^{1,2}$, Omar Mossad $^{1,2}$, Ori Staszewski ${ }^{1}$, Jana Neuber ${ }^{1,2}$, Bahtiyar Yilmaz ${ }^{3}$, Daniel Schnepf ${ }^{4}$, Mercedes Gomez de Agüero ${ }^{3}$, Stephanie C. Ganal-Vonarburg ${ }^{3}$, Andrew J. Macpherson ${ }^{3}$, Melanie Meyer-Luehmann ${ }^{5,6}$, Peter Staeheli ${ }^{4}$, Thomas Blank', Marco Prinz ${ }^{1,6,7}$ and Daniel Erny ${ }^{1,8^{*}}$ (D)

\begin{abstract}
It was recently revealed that gut microbiota promote amyloid-beta $(A \beta)$ burden in mouse models of Alzheimer's disease (AD). However, the underlying mechanisms when using either germ-free (GF) housing conditions or treatments with antibiotics (ABX) remained unknown. In this study, we show that GF and ABX-treated $5 \times$ familial $A D$ (5XFAD) mice developed attenuated hippocampal $A \beta$ pathology and associated neuronal loss, and thereby delayed disease-related memory deficits. While A $\beta$ production remained unaffected in both GF and ABX-treated 5XFAD mice, we noticed in GF 5XFAD mice enhanced microglial $A \beta$ uptake at early stages of the disease compared to ABX-treated 5XFAD mice. Furthermore, RNA-sequencing of hippocampal microglia from SPF, GF and ABX-treated 5xFAD mice revealed distinct microbiota-dependent gene expression profiles associated with phagocytosis and altered microglial activation states. Taken together, we observed that constitutive or induced microbiota modulation in 5xFAD mice differentially controls microglial A $\beta$ clearance mechanisms preventing neurodegeneration and cognitive deficits.
\end{abstract}

Keywords: Gut microbiota, Antibiotics, Germ-free, Microglia, Alzheimer's disease

\section{Introduction}

Alzheimer's disease (AD) is the most common neurodegenerative disease and cause of dementia worldwide [9]. The progressive loss of neurons causes symptoms such as memory loss and cognitive decline. Despite enormous efforts in $\mathrm{AD}$ research, the etiology of the frequent sporadic form of AD remains largely unknown [47]. The hereditary familial form of AD accounts only for less than

\footnotetext{
* Correspondence: daniel.erny@uniklinik-freiburg.de

'Institute of Neuropathology, University of Freiburg, Breisacher Str. 64, 79106 Freiburg, Germany

${ }^{8}$ Berta-Ottenstein-Programme, Faculty of Medicine, University of Freiburg, Freiburg, Germany

Full list of author information is available at the end of the article
}

$5 \%$ of all cases of $\mathrm{AD}$ and typically has a much earlier onset [51]. The main hallmarks of this incurable disease in the brain are extracellular amyloid-beta $(\mathrm{A} \beta)$ depositions and intracellular neurofibrillary tangles. The early aggregation and cerebral deposition of $A \beta$ is suggested to take place decades before the first symptoms appear [48], and $A \beta$ depositions mainly drive the development and progression of $\mathrm{AD}$ [53]. In the central nervous system $(\mathrm{CNS})$ microglia represent the main innate immune cells, and in contrast to other tissue macrophages have a unique solely yolk-sac origin $[17,24,46]$. While they are essential for maintaining tissue homeostasis during physiological conditions [25], microglia continuously survey their microenvironment, react to pathological 
stimuli and are involved in virtually all CNS diseases including AD pathogenesis [39, 44]. Recent genome-wide association studies (GWAS) showed that genes mainly or exclusively expressed by microglia, including complement receptors, or triggering receptor expressed on myeloid cells-2 (TREM2) are associated with increased risk of developing AD [28]. Microglia sense A $\beta$ by several receptors (i.e. TREM2) resulting in microglial activation, accumulation around $A \beta$ plaques and production of potential neurotoxic proinflammatory cytokines [44, 59]. As the professional phagocytes of the CNS, microglia play a crucial role in the removal of $A \beta$ aggregates [44]. Interestingly, microglia in Trem2-deficient 5xFAD mice incorporated less $A \beta$ than microglia from Trem2expressing 5xFAD mice [60].

Growing body of evidence highlight a considerable influence of gut microbes on the host's health [21]. Microbiota-derived molecules, such as short-chain fatty acids (SCFAs), have been shown to contribute to host's physiology and boost immune functions [21]. We have previously demonstrated that the maturation and function of microglia were highly affected by host gut microbes [12]. In mice born and raised under germ-free (GF) conditions, microglia displayed an immature homeostatic phenotype defined by altered gene expression, increased numbers, and hyper-ramified morphology. In addition, they displayed compromised immune response upon acute challenge with bacterial molecules and virus infection [12]. Notably, temporal eradication of gut bacteria by antibiotic treatment $(\mathrm{ABX})$ in former colonized adult SPF mice induced a comparable microglial phenotype to GF mice, arguing that constant signals from gut bacteria are necessary for proper microglial homeostasis und function [12]. Remarkably, the pathophysiology of several CNS diseases including AD was recently linked to host microbiota [14]. Harach and colleagues uncovered that AD pathology is diminished in the double transgenic APPPS1 AD mouse model under GF housing conditions compared to colonized controls [18]. Furthermore, postnatal long-term microbiota manipulation by $\mathrm{ABX}$ reduced $\mathrm{A} \beta$ burden in $\mathrm{APP}_{\mathrm{SWE}} / \mathrm{PS}_{\triangle \mathrm{E} 9}$ mice compared to non-treated controls [34]. However, the precise role of microglia, the involved cellular mechanism, the impact on cognitive function, and whether the production or degradation of $A \beta$ is affected, remained unclear.

In order to gain mechanistic insights, we characterized the $\mathrm{A} \beta$-associated pathology and related hippocampusassociated behavior at an early and progressed stage of the disease using $5 \mathrm{x}$ familial $\mathrm{AD}$ (5xFAD) mice bred under both GF and specific pathogen free (SPF) conditions and induced gut bacteria depletion by $\mathrm{ABX}$ in a third cohort. Additionally, we thoroughly investigated the influence of the constant and induced depletion of gut bacteria on microglia-mediated effects during $A \beta$ pathology. We revealed that constitutive absence of gut microbiota in GF 5xFAD mice increased the microglial uptake of $A \beta$ deposits in the hippocampus, resulting in decreased $\mathrm{A} \beta$ burden, associated neuronal loss and retained hippocampus-associated memory function, without affecting the production of $\mathrm{A} \beta$. While $\mathrm{ABX}$ induced acute depletion of gut bacteria resulted in similar decrease of $A \beta$ depositions, this effect was not attributed to microglial $A \beta$ phagocytosis.

\section{Materials and methods \\ Mice}

As a mouse model of Alzheimer's disease we used heterozygous male $5 x F A D$ transgenic mice and nontransgenic littermates ( 4 and 10 months of age) on a C57BL/6 J background co-expressing human

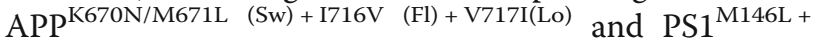
L286V under the control of the neuron-specific Thy-1 promoter [38]. Mice were housed under specific pathogen-free (SPF) conditions under a 12-h light, 12-h dark cycle with food and water ad libitum at CEMT (Freiburg, Germany). GF 5xFAD mice were generated via embryo transfer by Kathleen McCoy. GF heterozygous $5 \mathrm{xFAD}$ and WT littermates were obtained from the Clean Mouse Facility (Bern, Switzerland). In order to deplete microbiota, mice were treated orally via drinking water with a mixture of antibiotics (ABX), containing 1 $\mathrm{mg} / \mathrm{ml}$ vancomycin (Hexal), $1 \mathrm{mg} / \mathrm{ml}$ cefoxitin (Santa Cruz Biotechnology), $1 \mathrm{mg} / \mathrm{ml}$ gentamicin (Sigma-Aldrich) and $1 \mathrm{mg} / \mathrm{ml}$ metronidazol (Sigma-Aldrich) for 2 months as described previously [12]. All animal experiments were approved by the Ministry for Nature, Environment and Consumers' Protection of the state of Baden-Württemberg and were performed in accordance to the respective national, federal, and institutional regulations (G19-02 and X16-04A).

\section{Histology \& Immunofluorescence}

Mice were deeply anesthetized by intraperitoneal injection of a mixture of ketamine $(100 \mathrm{mg} / \mathrm{kg}$ body weight $)$ and xylazine $(10 \mathrm{mg}$ per $\mathrm{kg}$ body weight) and transcardially perfused with ice-cold 1xPBS. Collected brains were post-fixed in $4 \%$ PFA for $24 \mathrm{~h}$. One hemisphere was cryopreserved in 30\% sucrose for $48 \mathrm{~h}$, whereas the other hemisphere was paraffin embedded. Cryopreserved brains were frozen on dry ice and cut into $25 \mu \mathrm{m}$ thick coronal serial sections on a sliding microtome (SM2000R, Leica Biosystems) and collected in 5\% glycerol. As described previously [66], every tenth brain sections containing hippocampus from rostral to caudal were immunolabeled free-floating by incubating with anti-Iba1 antibody (rabbit, 1:500, Wako) and anti-Clec7a (1:30, Invivo Gen) for $24 \mathrm{~h}$ at $4{ }^{\circ} \mathrm{C}$. Subsequently, 
sections were incubated with Alexa-Fluor-488 and 647conjugated secondary antibody (1:500, Life technologies) for $24 \mathrm{~h}$ at $4{ }^{\circ} \mathrm{C}$. Compact $\mathrm{A} \beta$ plaques were stained with $2 \mu \mathrm{M}$ thiazine red (TR) (Sigma-Aldrich), a fluorescent congo red derivate, for $5 \mathrm{~min}$ at room temperature (RT). Finally, nuclei were stained with DAPI (4',6-diamidin-2phenylindol, 1:10000) for $10 \mathrm{~min}$ at RT. Additional series of brain sections were immunolabeled to visualize compact $A \beta$-plaques, as well as $1-16 \mathrm{~A} \beta$ precursor forms by using anti-6E10 antibody (1:1000, Biolegend) and antiIbal antibody $\left(1: 500\right.$, Wako) at $4{ }^{\circ} \mathrm{C}$ for $24 \mathrm{~h}$, followed by incubation with corresponding conjugated secondary antibodies Alexa-Fluor-568 and 488 (1:500, Life technologies) for $24 \mathrm{~h}$ at $4{ }^{\circ} \mathrm{C}$. DAPI was used as a nuclear stain for $10 \mathrm{~min}$ at RT. A $\beta$-plaque load and density of Iba1-immunoreactive cells were quantified throughout the entire hippocampus. In total 10 to 12 sections were analyzed per mouse.

Paraffin-embedded brains were cut sagittal into $3 \mu \mathrm{m}$ thin sections and immunofluorescence labeling was performed by using following primary antibodies: antiNeuN (1:200, Abcam), anti-Iba1 (1:500, Synaptic System), anti-P2ry12 (1:200, AnaSpec) and anti-ApoE (1:50, Merck), upon antigen retrieval $\left(\mathrm{pH} 6.0\right.$ citrate) at $4{ }^{\circ} \mathrm{C}$ for $24 \mathrm{~h}$. Subsequently, respective conjugated secondary antibodies Alexa-Fluor 647 or 488 (1:500, Life technologies) were used for $2 \mathrm{~h}$ at RT. A $\beta$ plaques were visualized by TR for $5 \mathrm{~min}$ at RT. Nuclei were stained with DAPI (1:10000) for $10 \mathrm{~min}$ at RT. Fluorescence images were taken with BZ-9000 Biorevo microscope (Keyence). Sections were analyzed by using BZ-II Analyzer Software (Keyence). Confocal images were taken with Olympus Fluoview FV 1000 confocal laser scanning microscope (Olympus).

\section{Behavior analysis}

Spatial working memory was tested using the continuous spontaneous alternation task in a T-maze as described previously $[10,49]$. In short, animals were set into the base of a T-maze and forced to explore one of the Tmaze arms until it returned to the base arm. Subsequently, the blocked arm was opened, and the animal could explore the maze. Once one arm of the T-maze was entered, the other arm was blocked until the animal returned to the base arm. Then, the exit of the base arm was blocked for $5 \mathrm{~s}$ and the animal explored the maze again. The experiment was stopped after 14 free choice arm entries. Arm entries were scored as alternations if an animal chose the opposing arm compared to the arm visited immediately prior to the scored instance. Repetitive arm entries were scored as entering the same arm for the third or more consecutive time. The total time after 14 free choice arm entries was recorded.
The Novel Object Recognition (NOR) task was applied to evaluate recognition memory as published previously $[2,37]$. During the habituation phase, each mouse could explore two similar objects within a total exploration time of $20 \mathrm{~s}$. We commenced the testing phase $6 \mathrm{~h}$ after the habituation phase. During the testing session, each mouse could explore a familiar object and a novel object of different shape and texture. The position of the novel object and the familiar object was randomized between each mouse. The time spent by each mouse to explore the novel object and the familiar object was noted. The experiment was stopped when the total exploration time reached $20 \mathrm{~s}$. The time spent by each mouse to explore the novel object and the familiar object, as well as the total time for paradigm completion during the testing phase was recorded.

\section{Ex vivo microglia isolation and flow cytometry}

Hippocampi were collected and microglia were enriched by using density gradient separation and were prepared as described previously [12, 65]. The cell suspension was then incubated with Fc receptor blocking antibody CD16/CD32 (1:200, BD Bioscience) and Fixable Viability Dye eFluor 780 (1:1000, eBioscience) for $10 \mathrm{~min}$ at $4{ }^{\circ} \mathrm{C}$. Subsequently, the following antibodies were used: antiCD11b (1:200, clone M1/70, Biolegend), anti-CD45 (1:200, clone 30-F11, BioLegend), anti-CD11c (1:100, clone N418, Biolengend) and for lineage exclusion by a dump gate antiCD3 (1:300, clone 17A2, Biolegend), anti-CD19 (1:300, clone 6D5, Biolegend), anti-CD45R (1:300, clone RA3-6B2, BD Bioscience), anti-Ly6C (1:300, clone AL-21, BD Bioscience) and anti-Ly6G (1:300, clone 1A8, BD Bioscience) and incubated for $30 \mathrm{~min}$ at $4{ }^{\circ} \mathrm{C}$.

\section{Ex vivo $A \beta$ phagocytosis assay}

Mice were injected intraperitoneally with methoxy-X-O4 (Tocris) (10 mg/kg bodyweight), a fluorescent congo red derivate, in a DMSO/PBS mixture. After $3 \mathrm{~h}$, hippocampi were collected and microglial cells were assessed as described previously [65]. Percentage of methoxy-XO-4 positive microglia were determined by flow cytometry using a FACS Canto II (BD Bioscience) and analyzed with FlowJo software (Tree Star).

\section{Microbial profiling of caecal contents}

DNA extraction, 16S rRNA sequencing and computation analysis of caecal contents from 4 months old SPF and ABX-treated 5xFAD mice and respective WT controls was performed as described previously [62] with slight modifications. $12 \mathrm{SPF}$ and $16 \mathrm{ABX}$-treated $5 \mathrm{xFAD}$ mice, as well as $15 \mathrm{SPF}$ and $14 \mathrm{ABX}$-treated WT mice were analyzed. In brief, caecal contents were collected in $2 \mathrm{ml}$ microfuge tubes and stored at $-80^{\circ} \mathrm{C}$ prior to DNA extraction. Total DNA was isolated from samples using 
the QIAamp DNA stool kit (Qiagen) according to the modified manufacturer's instructions. Afterwards, 100 - $400 \mathrm{ng}$ of DNA samples were subjected to amplification of V5/V6 region of bacterial 16S rRNA. Bacteriaspecific primers (forward 5' CCATCTCATCCCTGCG TGTCTCCGACTCAGC barcode ATTAGATACC CYGGTAGTCC 3' and reverse 5' CCTCTCTATG GGCAGTCGGTGATACGAGCTGACGACARCCATG-3') were used. Amplicon sequencing was performed using the Ion PGM ${ }^{\mathrm{mw}}$ Sequencing 400 Kit and Ion $316^{\mathrm{mm}}$ Chip V2 within the Ion $\mathrm{PGM}^{\mathrm{m}}$ System (Thermo Fisher). Fastq sequencing files were first loaded into the QIIME 1.9.1 pipeline [8], using custom analysis scripts for analysis on the UBELIX Linux cluster of the University of Bern [62]. The biom file and mapping file were used for statistical analyses and data visualization in the $\mathrm{R}$ with package phyloseq. The $\alpha$-diversity (Observed OTUs, Simpson and Shannon index), $\beta$-diversity (Bray-Curtis genuslevel community dissimilarities), and statistical analysis of clustering using Mann-Whitney $U$ tests for alpha diversity and Adonis (PERMANOVA) for beta diversity to confirm that the strength and statistical significance of groups in the same distance metrics in phyloseq using $\mathrm{R}$ [33]. The multivariate analysis by linear models (MaAsLin) $\mathrm{R}$ package were used to find associations between metadata and microbial community abundance [36]. Plots were generated with ggplot2 using phyloseq object.

\section{Quantification of bacterial load by flow cytometry}

Fecal samples were collected from SPF, GF and ABXtreated mice and weighted, immediately homogenized in ice-cold 1xPBS and filtered through $50 \mu \mathrm{m}$ CellTrics filters (Sysmex). A fraction of the filtrates was diluted 1:20 in $1 \times$ PBS and centrifuged for $5 \mathrm{~min}$ at $3000 \mathrm{~g}$ at $4{ }^{\circ} \mathrm{C}$. Subsequently, supernatant was aspirated and Syto9 (1:1000 in PBS, Thermo Fisher), a dye to identify gram + and gram- bacteria was added for $10 \mathrm{~min}$ at $4{ }^{\circ} \mathrm{C}$. DAPI $(1: 1000)$ was used for dead cell exclusion and the percentage of live bacteria was recorded by using flow cytometry. Flow cytometry cell counting beads (1:20, Thermo Fisher) were added to quantify absolute number of live bacteria per $\mathrm{mg}$ fecal sample.

\section{RNA-sequencing}

Total RNA was extracted from FACS sorted viable $\mathrm{CD}_{11} \mathrm{~b}^{+} \mathrm{CD} 45^{\text {low }} \mathrm{DUMP}^{-}$hippocampal microglia cells using the ARCTURUS ${ }^{\bullet}$ PicoPure $^{\oplus}$ RNA Isolation Kit (Thermo Fisher) according to manufacturer's protocol. The SMARTer Ultra Low Input RNA Kit for Sequencing v4 (Clontech Laboratories, Inc., Mountain View, CA, USA) was used to generate first strand cDNA from 300 pg total-RNA. Double stranded cDNA was amplified by
LD PCR (13 cycles) and purified via magnetic bead clean-up. Library preparation was carried out as described in the Illumina Nextera XT Sample Preparation Guide (Illumina, Inc., San Diego, CA, USA). 150 pg of input cDNA were tagmented (tagged and fragmented) by the Nextera XT transposome. The products were purified and amplified via a limited-cycle PCR program to generate multiplexed sequencing libraries. For the PCR step 1:5 dilutions of index 1 (i7) and index 2 (i5) primers were used. The libraries were quantified using the KAPA SYBR FAST ABI Prism Library Quantification Kit (Kapa Biosystems, Inc., Woburn, MA, USA). Equimolar amounts of each library were pooled, and the pools were used for cluster generation on the cBot with the Illumina TruSeq SR Cluster Kit v3. The sequencing run was performed on a HiSeq 1000 instrument controlled by the HiSeq Control Software (HCS) 2.2.38, using the indexed, 50 cycles singleread (SR) protocol and the TruSeq SBS v3 Reagents according to the Illumina HiSeq 1000 System User Guide. Image analysis and base calling were done by the Real Time Analysis Software (RTA) 1.18.61. The resulting .bcl files were converted into fastq files with the CASAVA Software 1.8.2. Library preparation and RNAseq were performed at the Genomics Core Facility "KFB - Center of Excellence for Fluorescent Bioanalytics" (University of Regensburg, Regensburg, Germany; www.kfb-regensburg.de).

Fastq files were quality controlled using FastQC [1] and reads were mapped to the GRCm38 mouse genome using the Star aligner [11]. Read counts were obtained using the featureCounts program [30] in conjunction with the Gencode transcriptome version M21 [15]. Differential gene expression analysis was performed using the limma/voomWithQualityWeights pipeline in $\mathrm{R}[29$, 31]. Venn diagram was generated by using previously published tools [19]. Heatmaps were generated using the package pheatmap [40]. Pathway analysis was performed using Ingenuity Pathway Analysis (IPA, QIAGEN).

\section{Elisa}

Quantification of soluble and insoluble $A \beta 40$ and 42 species in hippocampal homogenates were quantified by performing enzyme-linked immunosorbent assay as described previously [66]. Briefly, hippocampi were harvested and homogenized $(10 \% \mathrm{w} / \mathrm{v})$ in $1 \mathrm{xPBS}$ containing protease inhibitor and sequentially extracted with PBS (soluble fraction), $\mathrm{PBS}+0.1 \%$ Triton X-100 (membrane bound fraction) and finally with $8 \mathrm{M}$ guanidine hydrochloride solution (insoluble fraction). Protein concentration in each fraction was measured by using Bradford assay (Carl Roth) and ELISA was performed using $\mathrm{Hu}$ man $A \beta 42$ ultrasensitive ELISA kit and Human A $\beta 40 /$ A 342 ELISA kits (Invitrogen) according to manufacturer's protocols. 


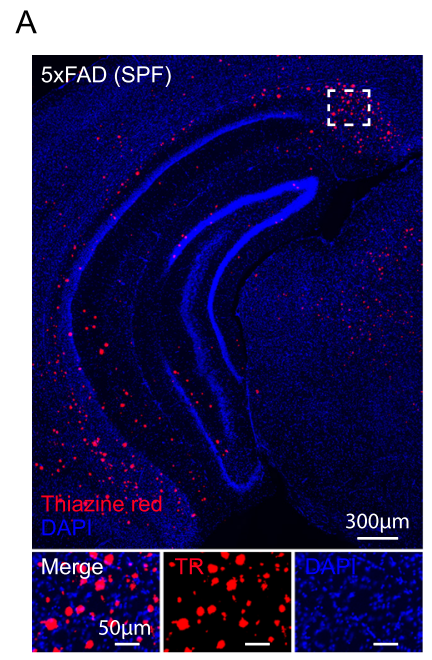

E $\quad$ SPF $\square G F \circ A B X F$

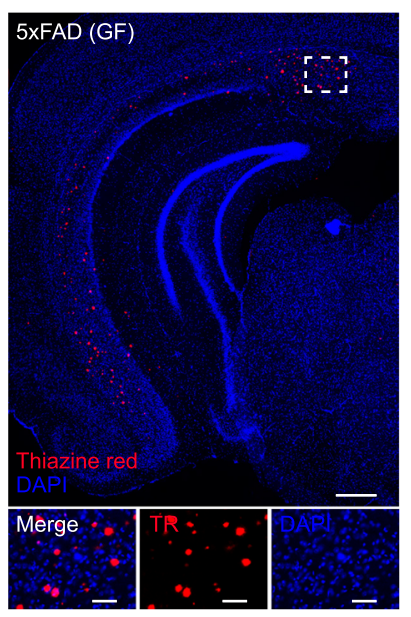

G

$\mathrm{H}$

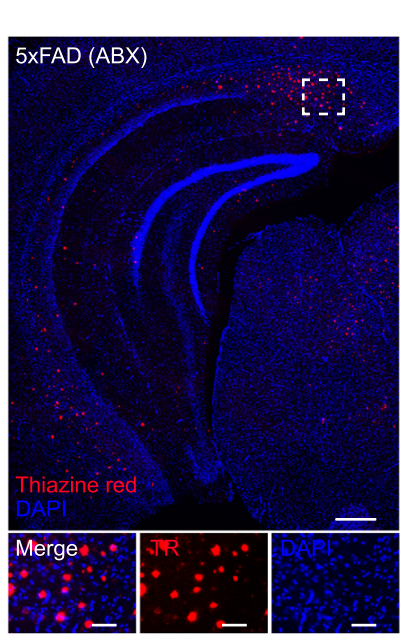

I

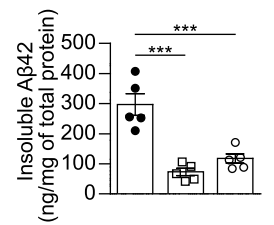

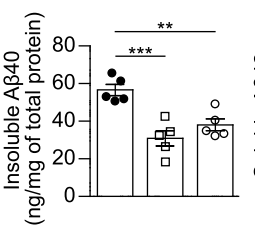

$\mathrm{J} \quad$ SPF $\triangle \mathrm{GF} \circ \mathrm{ABX} \mathrm{K}$

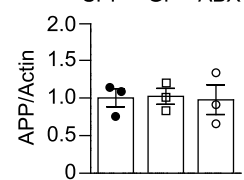

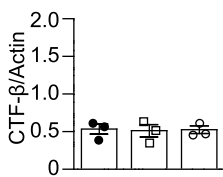

$\mathrm{N} \cdot \mathrm{SPF} \square \mathrm{GF} \circ \mathrm{ABX} \mathrm{O}$ $\widehat{\overparen{E}}_{1000}$ * 800 *** 这远 $800-$ -

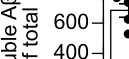
के

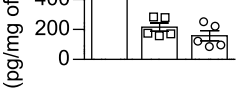

$\mathrm{L}$

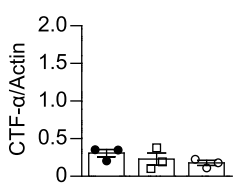

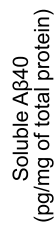

$\mathrm{M}$

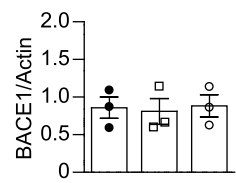

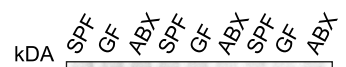

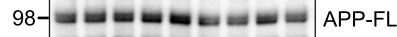
14- CTF- $\beta$

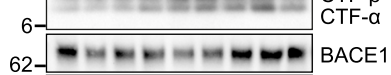

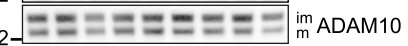

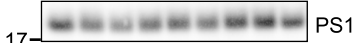

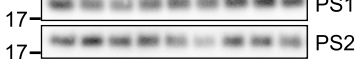
$14-\ldots$ PEN2

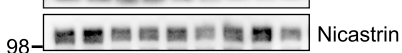
$98-\ldots A B$ B8-Actin P Q $\mathrm{R}$ $\mathrm{S}$

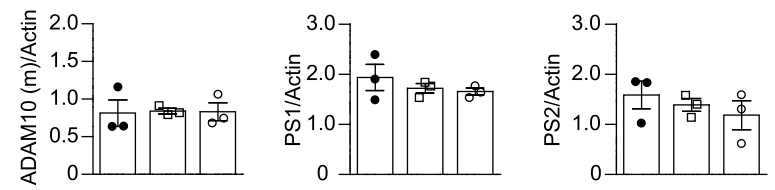

$\mathrm{T}$
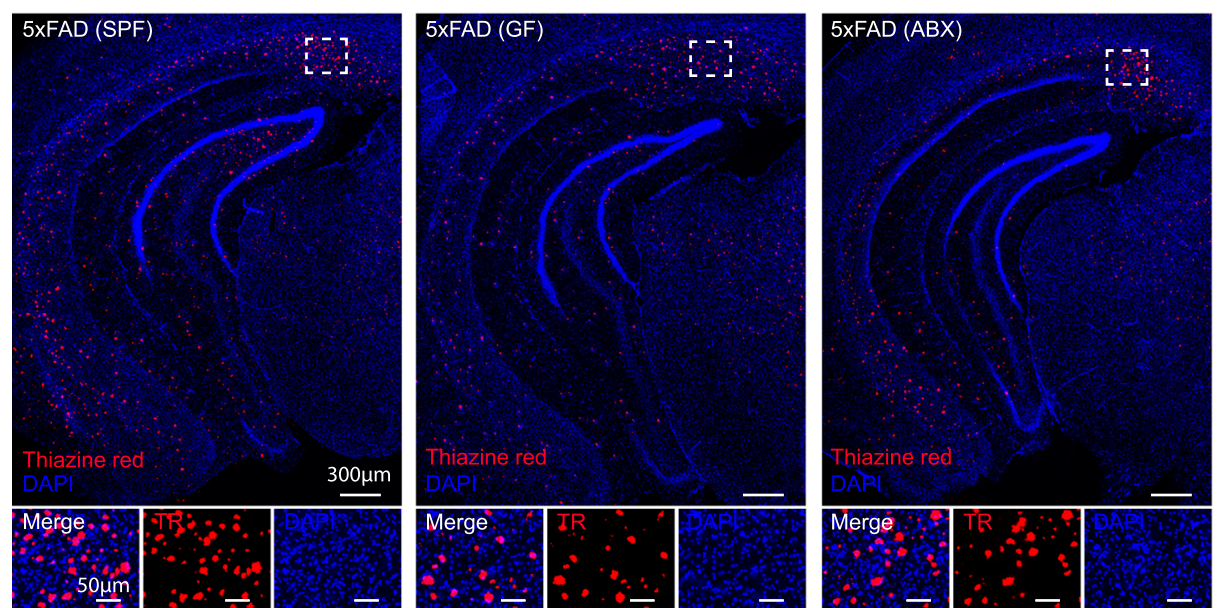

U

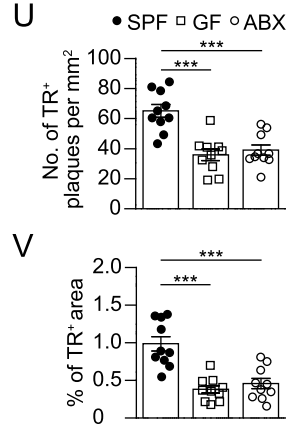

$\mathrm{V}$

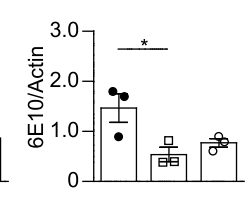
B SPF $\square$ GF $\circ A B X$

D
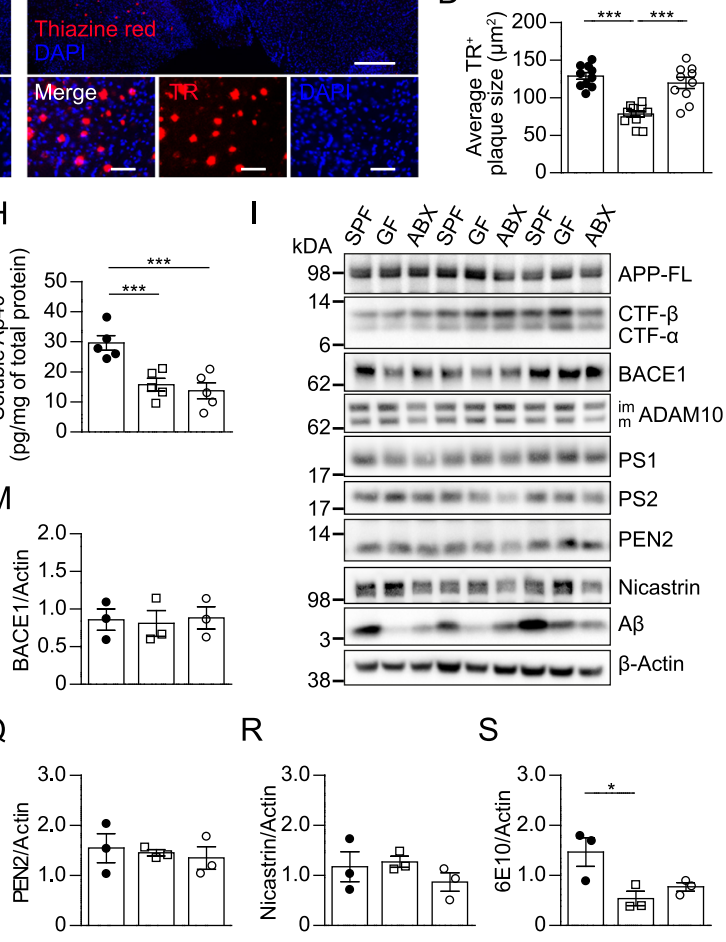

W

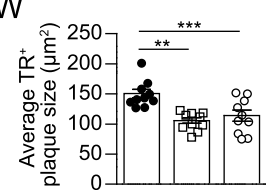

Fig. 1 (See legend on next page.) 
(See figure on previous page.)

Fig. 1 Absence of host microbiota reduces hippocampal A $\beta$ depositions of 5XFAD mice. (a) Representative fluorescence images of thiazine red ${ }^{+}$ (TR; red) compact AB plaques in the hippocampus of 4 months old SPF, GF and ABX-treated 5xFAD mice. Nuclei were stained with DAPI (blue). Overview of hippocampus and magnification of subiculum (dashed line) are shown. Scale bars represent $300 \mu \mathrm{m}$ (overview) and $50 \mu \mathrm{m}$ (insert). (b) Quantification of the number of $\mathrm{TR}^{+} \mathrm{A} \beta$-plaques per $\mathrm{mm}^{2}$, (c) percentage of $\mathrm{TR}^{+}$area and (d) average $\mathrm{TR}^{+}$plaque size $\left(\mu \mathrm{m}^{2}\right)$ in coronal hippocampal sections. Each symbol represents one mouse. Data are presented as mean \pm s.e.m. Significant differences were determined by oneway ANOVA followed by Tukey's post-hoc comparison test ( ${ }^{*} P<0.05$, ${ }^{* *} P<0.01$, $\left.{ }^{* *} P<0.001\right)$. Data are representative of four independent

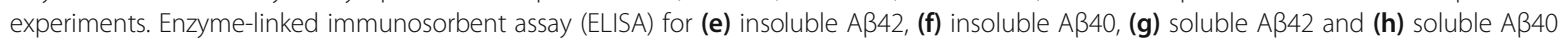
fractions of hippocampal brain extracts from 4 months old SPF, GF and ABX-treated 5xFAD mice. Each symbol represents one mouse. Data are presented as mean \pm s.e.m. Significant differences were determined by one-way ANOVA followed by Tukey's post-hoc comparison test $(* * P<0.01$, $\left.{ }^{* * *} \mathrm{P}<0.001\right)$. Data are representative of two independent experiments. (i) Representative immunoblots of hippocampal brain homogenates from 4 months old SPF, GF and ABX-treated 5xFAD mice against human full-length amyloid precursor protein (APP-FL), C-terminal fragment (CTF) a, CTF- $\beta, \beta$-site of APP cleaving enzyme (BACE) 1, A Disintegrin And Metalloproteinase (ADAM10), $\gamma$-secretase complex (Nicastrin, presenilin enhancer (PEN) 2, Presenilin (PS) 1, PS2) and AB (6E10). $\beta$-Actin was used as loading control. Each lane represents one mouse. Quantification of (j) APP-FL, (k), CTF- $\beta$, (I) CTF-a, (m) BACE1, (n) ADAM10, (o) PS1, (p) PS2, (q) PEN2, (r) Nicastrin, and (s) A $\beta$ protein levels normalized to $\beta$-Actin are shown. Each symbol represents one mouse. Data are presented as mean \pm s.e.m. Significant differences were determined by one-way ANOVA followed by Tukey's post-hoc comparison test ( $\left.{ }^{*} \mathrm{P}<0.05\right)$. Data are representative of two independent experiments. (t) Representative fluorescence images of $\mathrm{TR}^{+}$compact $\mathrm{A} \beta$ plaques in the hippocampus of 10 months old 5xFAD mice. Nuclei were stained with DAPI (blue). Overview of hippocampus and magnification of subiculum (dashed line) are shown. Scale bars represent $300 \mu \mathrm{m}$ (overview) and $50 \mu \mathrm{m}$ (insert). (u) Quantification of the number of $\mathrm{TR}^{+} \mathrm{A} \beta$-plaques per area $\left(\mathrm{mm}^{2}\right),(\mathbf{v})$ percentage of $\mathrm{TR}^{+}$area and $(\mathbf{w})$ average of $\mathrm{TR}^{+}$plaque size $\left(\mu \mathrm{m}^{2}\right)$. Each symbol represents one mouse. Data are presented as mean \pm s.e.m. Significant differences were determined by one-way ANOVA followed by Tukey's post-hoc comparison test $\left({ }^{* *} P<0.01,{ }^{* * *} P<0.001\right)$. Data are representative of four independent experiments

\section{Western blot analysis}

Hippocampi were harvested and homogenized in RIPA buffer (25 mM Tris- $\mathrm{HCl}, 150 \mathrm{mM} \mathrm{NaCl}, 1 \%$ Nonidet P-40, 0.5\% sodium deoxycholate, 0.1\% SDS, protease inhibitor, $\mathrm{pH} 7.5$ ) to extract total protein. Total protein concentration was determined by using Bradford assay (Carl Roth). Samples were separated by $4-12 \%$ NuPAGE Bis-tris mini gels (Invitrogen) and immunoblotted using antibodies against APP and CTFs (1:3000, Sigma), BACE1, ADAM10 (1:1000, Cell Signaling Technology), Nicastrin, PEN2, Presenilin 1 and 2 (PS1, PS2) (1:1000, $\gamma$ Secretase Antibody Sampler Kit, Cell Signaling Technology) and anti-A $\beta$ (6E10, 1:3000, Biolegend) for $24 \mathrm{~h}$ at $4{ }^{\circ} \mathrm{C}$. Anti- $\beta$ actin-HRP (1:5000, Abcam) was used as loading control. Immunoblots were incubated with corresponding HRP-linked secondary antibodies for $1 \mathrm{~h}$ at RT and visualized by using SuperSignal ${ }^{\mathrm{TM}}$ West Femto Maximum Sensitivity Substrate (Thermo Fisher).

\section{Statistical analysis}

Statistical analysis was performed using GraphPad Prism (GraphPad Software, Version 5.0, La Jolla, USA). All data were tested for normality applying the Shapiro-Wilk normality test. If normality was given, either an unpaired $t$ test, one-way ANOVA followed by Tukey's post-hoc comparison test or two-way ANOVA followed by Bonferroni's compression was applied respectively. Differences were considered significant when $P$ value $<0.05$. To obtain unbiased data, experimental mice were all processed together. Cell quantifications were performed blinded by two scientists independently and separately.

\section{Results}

\section{Absence of gut microbiota ameliorates hippocampal $A \beta$ burden of 5xFAD mice}

To investigate whether gut microbiota is detrimental or beneficial during neurodegeneration, we took advantage of the $5 x F A D$ mouse model that recapitulates major features of the $A \beta$ pathology [38]. In 5xFAD mice, $A \beta$ plaques start to appear at around 2 months of age [38]. In order to evaluate the modulation of an early phase of the disease in $5 x F A D$ mice by gut microbiota, we first compared 4 months old GF 5xFAD to colonized (specific pathogen free, SPF) 5xFAD mice. Additionally, we treated SPF 5xFAD mice orally with ABX for 2 months prior to analyze at an age of 4 months.

GF-housed and ABX-treated mice displayed enlarged caeca with dark-colored caecal contents and incremented caecal weights compared to SPF controls, as previously described [12] (Suppl. Fig. 1A-C). To confirm the successful reduction of gut bacteria after ABX application, we quantified the number of live bacteria in fecal samples by flow cytometry (Suppl. Fig. 1D - F). In line, we observed a significant reduction in species richness in ABX-treated 5xFAD mice and wild-type (WT) littermates (Suppl. Fig. $1 G \& \mathrm{H}$ ). In contrast to a recent report [5], we did not detect substantial differences in species richness (q-value <0.05) in caecal contents of SPF 5xFAD and non-transgenic littermates (Suppl. Fig. $1 \mathrm{I}-\mathrm{K}$ and Additional files 6 and 7). First, to analyze the amount of $\mathrm{A} \beta$ depositions by histopathological assessment in hippocampi of 4 months old SPF, GF and ABX-treated 5xFAD mice, we took advantage of the fluorescent congo red derivate thiazine red (TR) which visualizes compact $A \beta$ depositions (Fig. 1A). In line with previous observations in other $\mathrm{AD}$ mouse models $[18,34]$, the amount of $\mathrm{TR}^{+}$ 


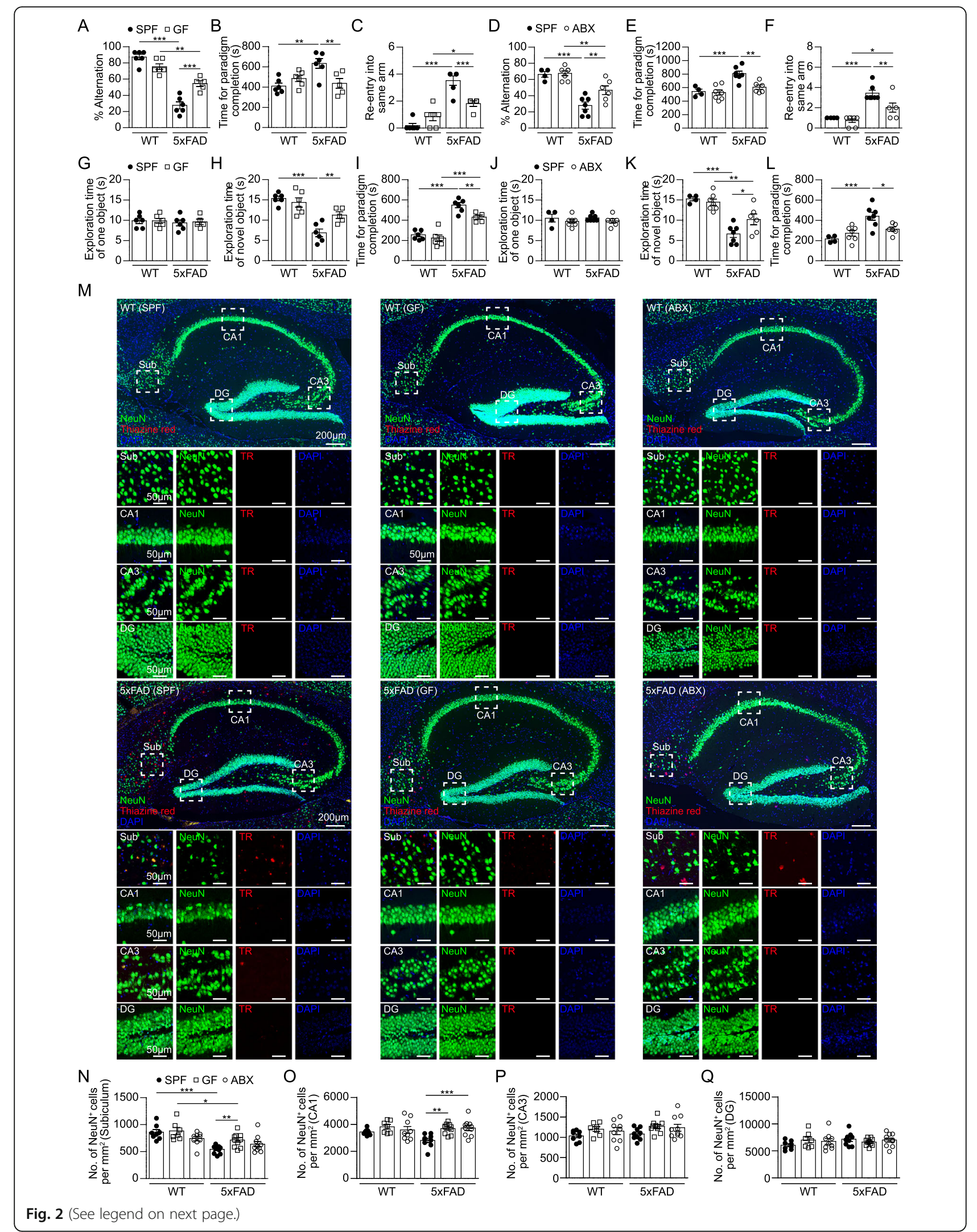


(See figure on previous page.)

Fig. 2 Restored memory deficits in 5xFAD mice lacking microbes. (a-c) T-maze test performance of 10 months old SPF and GF 5xFAD mice, as well as aged-matched WT controls or (d-f) of 10 months old SPF and ABX 5XFAD mice, as well as WT controls. (g-i) Novel object recognition test (NOR) of 10 months old SPF and GF 5xFAD mice, as well as age-matched WT controls or (j-l) of 10 months old SPF and ABX 5xFAD mice, as well as respective WT controls. Each symbol represents one mouse. Data are presented as mean \pm s.e.m. Significant differences were determined by two-way ANOVA followed by Bonferroni's post-hoc comparison test ( ${ }^{*} \mathrm{P}<0.05,{ }^{* *} \mathrm{P}<0.01,{ }^{* * *} \mathrm{P}<0.001$ ). Data are representative of three independent experiments. (m) Representative immunofluorescence images of $\mathrm{NeuN}^{+}$neurons (green) and $\mathrm{TR}^{+}$(red) compact $\mathrm{A} \beta$ plaques in the subiculum (Sub), cornu ammonis (CA) 1, CA3 and dentate gyrus (DG) of the hippocampus of 10 months old SPF, GF and ABX-treated 5xFAD mice. Nuclei were stained with DAPI (blue). Overview of hippocampus and magnifications of subiculum, CA1, CA3 and DG (dashed lines) are shown. Scale bars represent $200 \mu \mathrm{m}$ (overview) and $50 \mu \mathrm{m}$ (inserts). Quantification of the number of $\mathrm{NeuN}^{+}$neurons per mm ${ }^{2}$ in the subiculum $(\mathbf{n})$, CA1 (o), CA3 (p) and DG (q) of sagittal hippocampal sections from SPF, GF and ABX-treated 5xFAD and age-matched WT mice. Each symbol represents one mouse. Data are presented as mean \pm s.e.m. Significant differences were determined by two-way ANOVA followed by Bonferroni's post-hoc comparison test $\left.{ }^{* *} \mathrm{P}<0.01,{ }^{* * *} \mathrm{P}<0.001\right)$. Data are representative of two independent experiments

compact plaques was diminished in hippocampi of GF and ABX-treated 5xFAD mice compared to SPF 5xFAD controls (Fig. 1A - C). Additional immunofluorescence labelling with the 6E10 antibody, targeting compact and diffuse $A \beta$, showed similar results (Suppl. Fig. 2A - C). Of note, we observed significantly smaller plaques under GF conditions compared to SPF and ABX-treated 5xFAD mice (Fig. 1D \& Suppl. Fig. 2D). In accordance, both insoluble A 342 and A $\beta 40$ (Fig. 1E \& F, Suppl. Fig. 2E) as well as soluble $A \beta 42$ and $A \beta 40$ fractions (Fig. 1G \& H, Suppl. Fig. 2F) were significantly decreased in hippocampal brain homogenates of GF and ABX 5xFAD mice when compared to SPF 5xFAD controls. To assess a potential effect of gut microbiota on the processing of the amyloid precursor protein (APP), we measured APP and its C-terminal fragments $(\mathrm{CTF}-\alpha, \mathrm{CTF}-\beta)$ as well as secretases involved in APP-processing, including $\beta$ secretase ( $\beta$-site of APP cleaving enzyme, BACE1), A Disintegrin And Metalloproteinase (ADAM) 10 and components of the $\gamma$-secretase complex (Nicastrin, PEN2, Presenilin1, Presenilin 2) in hippocampal tissue extracts by immunoblotting and detected no alterations (Fig. 1I - R). Consistent with the ELISA results, western blot analysis also demonstrated significantly lower A $\beta$ levels in both GF and ABX 5xFAD mice compared to SPF 5xFAD animals (Fig. 1S).

Next, we asked whether the A $\beta$ accumulation was modulated at a later progressed stage of AD pathology by either constant or induced microbial absence. Therefore, we investigated SPF and GF housed 5xFAD mice at an age of 10 months. Additionally, $A B X$ treatment of SPF 5xFAD mice was started 2 months prior to analysis at an age of 10 months. Compared to the 4 months old SPF 5xFAD mice, we noticed a robust increase of $\mathrm{TR}^{+}$ $\mathrm{A} \beta$ depositions in 10 months old SPF 5xFAD animals, whereas GF and ABX-treated 5xFAD mice developed significantly less and smaller $A \beta$ plaques (Fig. $1 \mathrm{~T}-\mathrm{W}$ ). Consequently, both insoluble and soluble $A \beta 42$ and A 340 fractions were decreased in hippocampi of GF and ABX 5xFAD mice compared to SPF 5xFAD controls (Suppl. Fig. 2G - K). APP processing also showed no significant alterations in the aged GF 5xFAD mice (Suppl. Fig. 2L - U). Altered A $\beta$ load was additionally detected by immunoblot and $6 \mathrm{E} 10$ immunofluorescence labelling (Suppl. Fig. 2V - Z), indicating a possible modulation of $A \beta$ pathology even at later stages of the disease. In sum, our findings highlight a disease-promoting role of gut bacteria during $\mathrm{A} \beta$ mediated neurodegeneration, whereas modulation thereof might improve the disease course.

\section{Gut bacteria influence memory function of 5xFAD mice}

To elucidate whether the reduced $A \beta$ plaque burden in 10 months old GF and ABX 5xFAD mice compared to the steadily colonized SPF $5 x$ FAD controls is affecting cognitive function, we first examined spatial working memory in the T-maze paradigm. As expected, SPF $5 x F A D$ mice showed decreased percentage of arm alternation and more re-entries into the same arm of the T-maze (Fig. 2A - C) compared to age-matched SPF WT littermates. Both GF housing (Fig. 2A - C \& Suppl. Fig. 3A) and oral ABX application (Fig. 2D - F \& Suppl. Fig. 3B) partially rescued this phenotype in $5 x F A D$ mice, whereas the SPF, GF and ABX WT controls showed no behavioral abnormalities. Furthermore, when tested in the novel object recognition (NOR) task, GF 5xFAD (Fig. 2G - I) as well as ABX-treated 5xFAD mice (Fig. 2J - L) spent significantly more time exploring the novel object compared to SPF 5xFAD mice, suggesting partially protected learning and recognition memory. Importantly, impairment of spatial and recognition memory in colonized SPF 5xFAD mice was accompanied by prominent neuronal loss in the subiculum (sub) as well as in the cornu ammonis (CA) 1 region, while we did not observe significant neuronal loss in the CA3 and dentate gyrus (DG) (Fig. 2M - Q). Microbiota manipulation at early disease stages in 4 months old $5 x F A D$ mice did not yet affect markedly hippocampusassociated memory functions (Suppl. Fig. 3C - P) or neuronal survival (Suppl. Fig. 4Q \& R). Collectively, our data show a detrimental role of microbiota in the $5 x F A D$ mouse model worsening spatial and recognition 


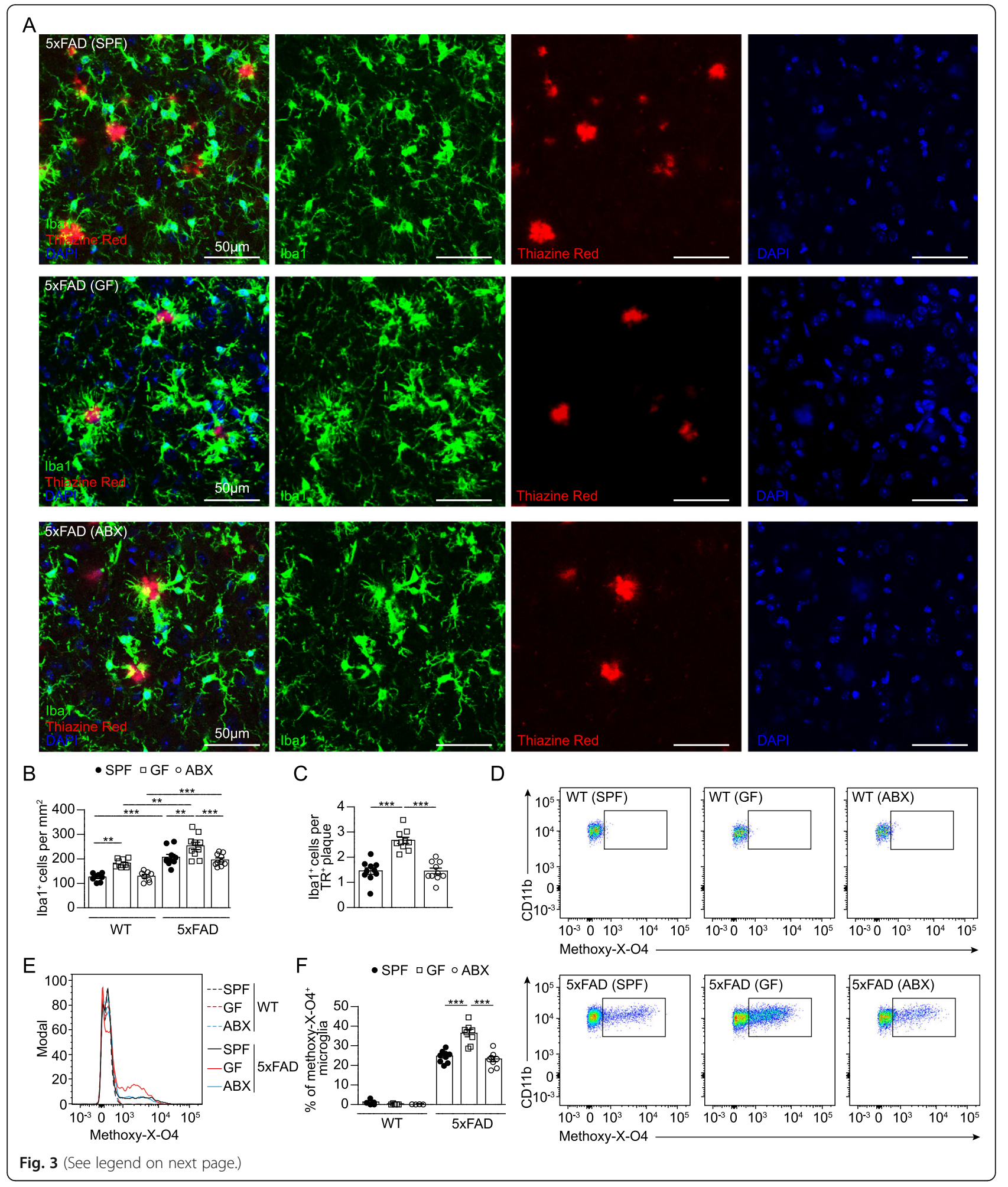




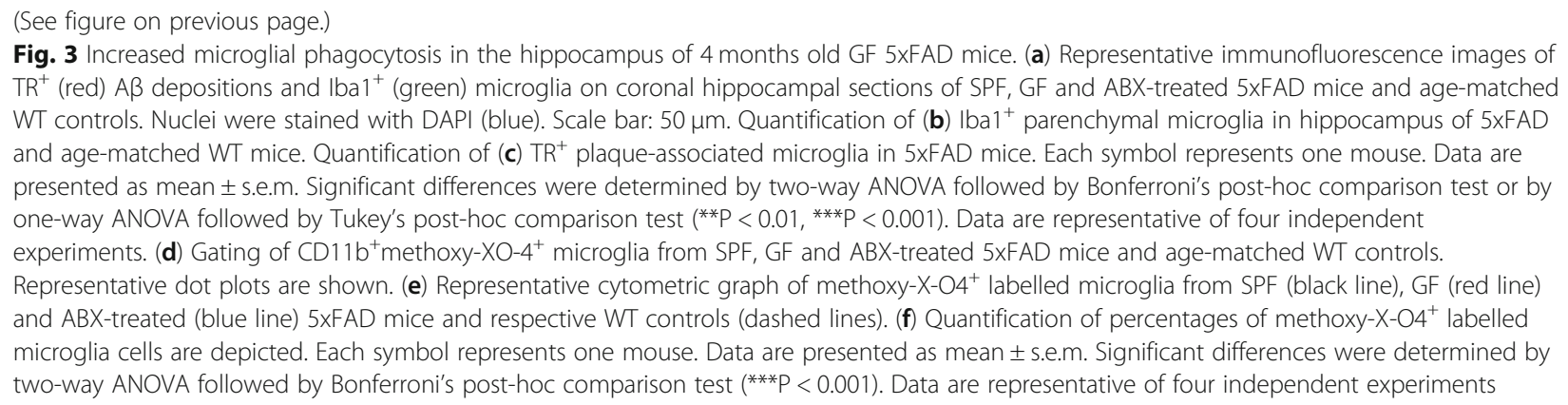

memory along with reduced neuronal numbers in the hippocampus.

\section{Constitutive lack of microbiota augments microglial uptake of $A \beta$ debris}

We previously demonstrated that microglial features are essentially steered by the host microbiota [12]. In addition, it is widely accepted that microglia critically contribute to $\mathrm{AD}$ progression [44]. Following our own and previous observations $[18,34]$ showing that microbiota have a negative impact on $\mathrm{AD}$ pathology in different mouse models of $\mathrm{AD}$, we aimed to clarify the cellular mechanism resulting in decreased $A \beta$ pathology under GF or ABX conditions. To examine the functional role of microglia in 4 months old SPF, GF and ABX-treated 5xFAD mice, we first quantified $\mathrm{Iba}^{+}$parenchymal microglia numbers in the hippocampi and compared them to the respective WT controls (Fig. 3A \& B, Suppl. Fig. 4A). $5 x F A D$ mice housed under GF conditions displayed an higher microglial density in the hippocampal tissue compared to SPF $5 x F A D$ mice, while ABX treatment did neither affect microglia density in 5xFAD mice nor WT mice in line with previous findings [12, 54] (Fig. 3A \& B, Suppl. Fig. 4A). Next, we examined in detail microglia located in a close proximity $(<10 \mu \mathrm{m})$ to $\mathrm{TR}^{+} \mathrm{A} \beta$ plaques. We noticed significantly more $\mathrm{TR}^{+} \mathrm{A} \beta$ plaque-associated $\mathrm{Iba}^{+}$ microglia in GF 5xFAD mice (Fig. 3A \& C), whereas SPF and ABX-treated 5xFAD mice showed less accumulation of $\mathrm{Ibal}^{+}$microglia around $\mathrm{TR}^{+} \mathrm{A} \beta$ plaques. Similar results were found by using $6 \mathrm{E} 10$ immunofluorescence for compact and diffuse $A \beta$ debris (Suppl. Fig. 4B \& C). It has been suggested that plaqueassociated microglia are critical in preventing senile $A \beta$ plaque formation in $\mathrm{AD}$ mouse models, for example by increased phagocytosis [23, 27]. Hence, we investigated whether permanent absence or induced microbiota depletion alters microglial phagocytosis by performing ex vivo flow cytometric based analysis of $A \beta$ uptake using methoxy-X-O4 staining as described before [65] (Fig. 3D $\&$ E, Suppl. Fig. $5 \mathrm{~A})$. In line with the increased $A \beta$ plaque- associated $\mathrm{Iba} 1^{+}$microglia numbers in GF $5 x F A D$ mice, we observed higher percentage of methoxy- $\mathrm{X}-\mathrm{O} 4^{+}$microglial cells under GF conditions (Fig. 3D - F), indicating higher $A \beta$ uptake. Notably, 2 months supplementation of $A B X$ via drinking water did not affect the percentage of methoxy-X-O $4^{+}$microglial cells which was comparable to SPF levels (Fig. 3D - F). These findings underline that $\mathrm{ABX}$-induced microbiota reduction in 5xFAD mice is not sufficient to modulate $A \beta$ uptake by microglia as observed in GF 5xFAD animals.

\section{Permanent absence of microbes alters microglial gene profile in GF 5xFAD mice}

To examine the effector functions of microglia in 5xFAD mice lacking microbes in more detail, we FACS-isolated hippocampal microglia from 4 months old SPF, GF and ABX 5xFAD mice and the respective age-matched WT controls and analyzed genome-wide mRNA expression profiles by RNA-sequencing (seq) (Fig. 4A \& B). In general, 680 genes were similarly modulated under SPF, $\mathrm{ABX}$ and GF conditions in 5xFAD mice compared with non-transgenic WT controls (Fig. 4A, Additional file 8). Notably, microglia from SPF 5xFAD animals regulated 1006 individual genes, whereas microglia from GF $5 x F A D$ mice displayed specific expression changes in 1185 genes and microglia in ABX-treated 5xFAD mice exhibited 700 uniquely transcribed gene transcripts (Fig. 4A). Statistical analysis of the most differently expressed genes across SPF and GF housing conditions and ABX treatment in WT and 5xFAD mice revealed 1945 significantly (adj. $P<0.0001$ ) up- (red) and downregulated (blue) genes in microglia (Fig. 4B, Additional file 9). Among the differently expressed genes, we observed in microglia from GF 5xFAD mice upregulated expression of apolipoprotein E (Apoe) and Trem2, which are implicated in $\mathrm{A} \beta$ detection and clearance $[44,60]$. Moreover, genes attributed to $\mathrm{AD}$-associated microglial activation $[23,27]$ including $A x l, C s t 7$, Itgax (encoding CD11c), $C d 9$ or Clec7a showed an overall increased and P2ry 12 reduced expression in microglia from GF $5 x$ FAD mice (Fig. 4B). Subsequent IPA pathway analysis of differentially expressed genes between microglia from WT and 
A

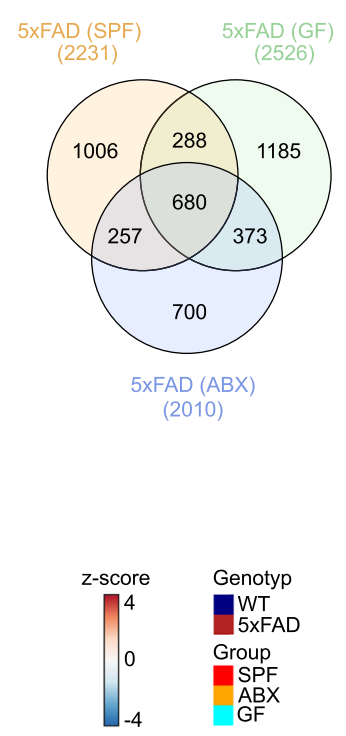

B
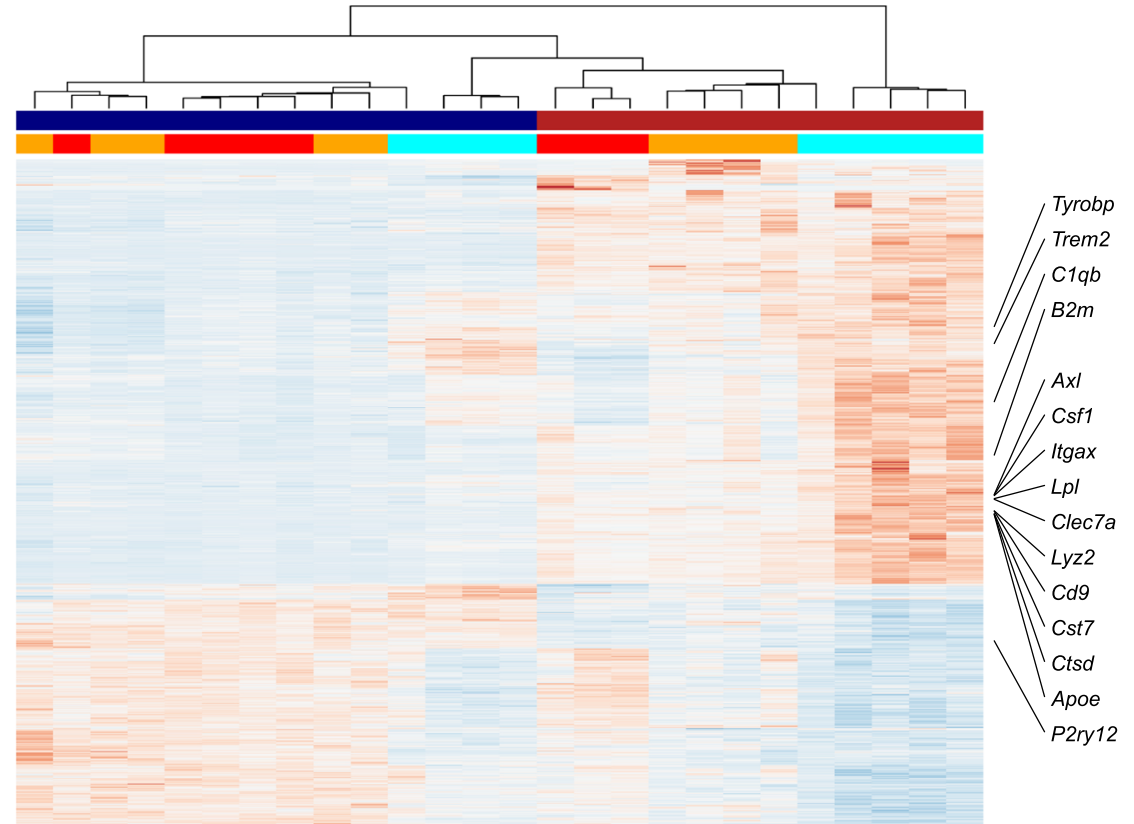

C

Pathways

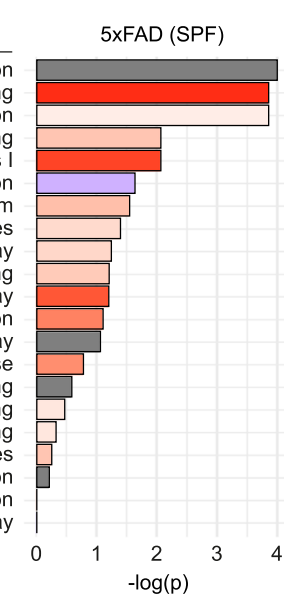

D
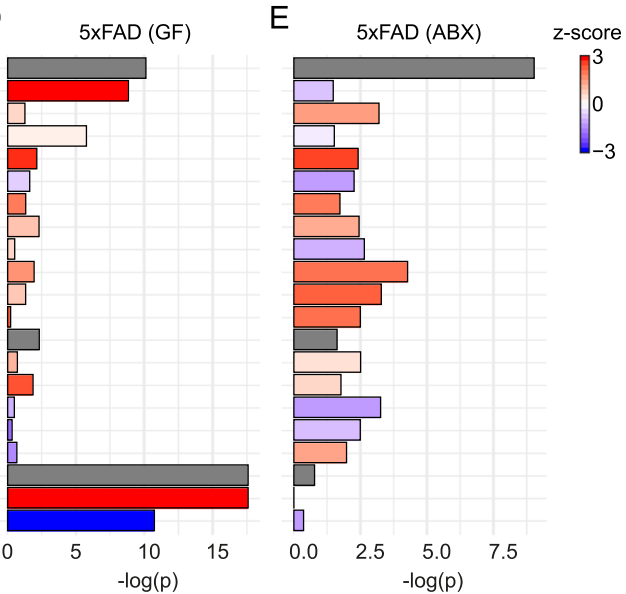
$\begin{array}{r}\text { mTOR Signaling } \\ \text { Glycolysis I } \\ \hline\end{array}$ Checkpoint Regulation Fc $\gamma$ R-mediated Phagocytosis in Macrophages and Monocytes STAT3 Pathway

Neuroinflammation Signaling Pathway Cyclins and Cell Cycle Regulation NRF2-mediated Oxidative Stress Response Cdc42 Signaling Chemokine Signaling Paxillin Signaling
Production of NO and ROS in Macrophages Mitochondrial Dysfunction Oxidative Phosphorylation

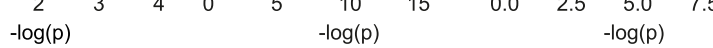

$\mathrm{F}$

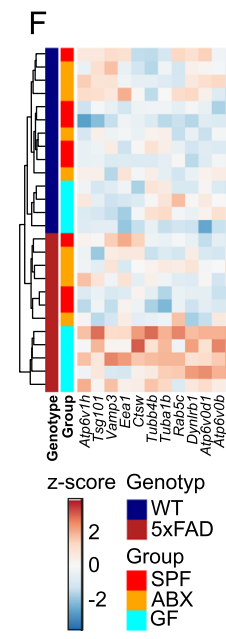

Fig. 4 (See legend on next page.) 


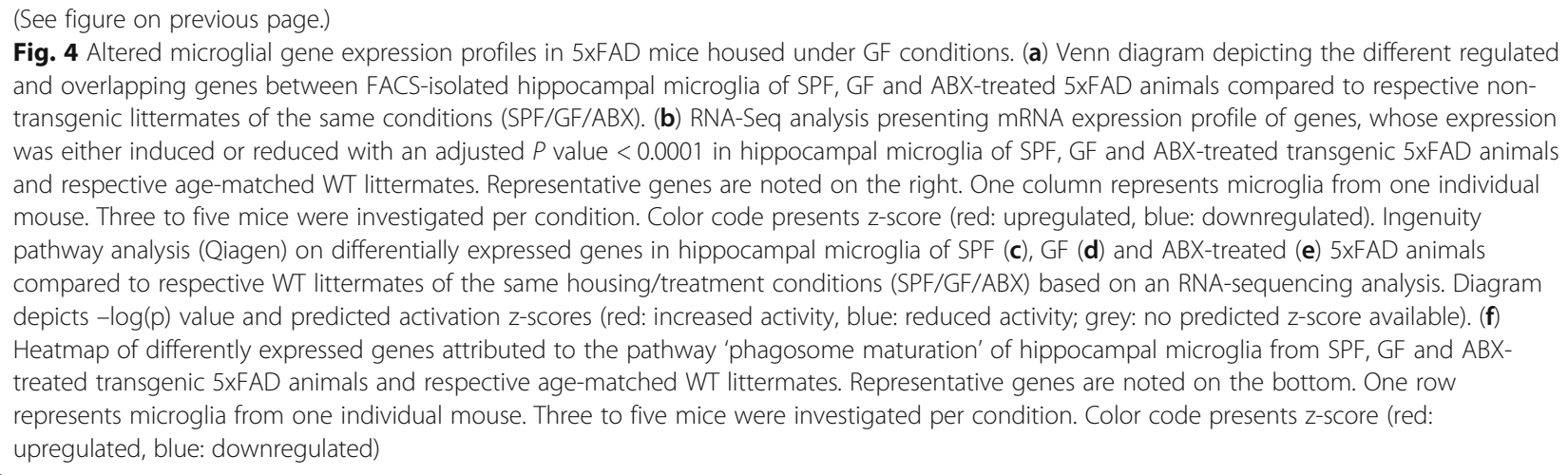

transgenic 5xFAD mice, housed under SPF or GF conditions or after $\mathrm{ABX}$ treatment, revealed strongly attenuated chemokine and mTOR signaling pathways in microglia from GF and ABX-treated 5xFAD mice, whereas neuroinflammation signaling pathways were robustly diminished in microglia from GF 5 xFAD mice, leading to a strongly diminished repertoire of innate immune response (Fig. 4C - E, Additional files 10, 11, and 12). In contrast, microglia in ABX-treated 5xFAD mice displayed increased activation $\mathrm{z}$-score of interferon signaling and Liver X Receptor (LXR) Retinoid X Receptor (RXR) activation, which belong to the type II family of nuclear receptors (NR). LXR and RXR signaling are involved in a wide range of $A \beta$-related effects and are also reported to promote anti-inflammatory effects in microglia [64]. Remarkably, we detected predominantly in microglia from GF 5xFAD mice robustly increased expression of genes attributed to oxidative phosphorylation and mitochondrial dysfunction, while the induction of genes linked to production of nitric oxide and reactive oxygen species were reduced concomitantly (Fig. 4D, Additional file 11). Further, we noticed an increased expression of genes implicated in phagocytosis, such as phagosome maturation and Cdc42 signaling [26], predominantly in microglia from GF $5 x F A D$ mice (Fig. 4C - F, Additional files 10, 11, 12). In sum, the data is indicating an altered activation state of microglia in GF 5xFAD mice, while microglial phagocytosis is preferentially enhanced under GF conditions.

Next, the differently expressed genes ApoE (Fig. 5A), P2ry12 (Fig. 5B), Clec7a (Fig. 5C) and Itgax (Fig. 5D) were verified on protein level. We noticed an increased percentage of $\mathrm{P} 2 \mathrm{ry} 12^{\mathrm{dim}} / \mathrm{Iba} 1^{+}$microglia in the hippocampus of GF $5 x F A D$ mice (Fig. $5 E \&$ F), whereby TR $^{+}$ plaque-associated microglia made up the majority of total P2ry12 ${ }^{\mathrm{dim}} / \mathrm{Iba1}^{+}$cells (Fig. 5G \& H). Furthermore, we confirmed increased $A p o E$ and $C l e c 7 a$ expression in hippocampal microglia from GF $5 x F A D$ mice as evident by increased percentages of $\mathrm{ApoE}^{+} / \mathrm{Ibal}^{+}$(Fig. 5I - L) and $\mathrm{Clec} \mathrm{a}^{+} / \mathrm{Iba}^{+}$plaque-associated and non-plaque- associated microglia (Fig. 5M - P). Additionally, we observed elevated expression of $\mathrm{CD}_{11 \mathrm{c}^{+}}$hippocampal microglia from GF $5 x F A D$ mice by flow cytometry (Fig. $5 \mathrm{Q}-\mathrm{S})$. Taken together, these findings demonstrate that the previously described AD-linked activation signature of microglia [23, 27] can be modulated by host microbiota.

\section{Microbiota-dependent enhanced microglial phagocytosis of $A \beta$ debris is age-related}

Since it is well acknowledged that microglia become exhausted during chronic exposure to $A \beta$ debris in mouse and human, we further examined whether microglial clearance capacity is sustained in later AD disease stage. First, we quantified $\mathrm{Iba1}^{+}$parenchymal microglia numbers in the hippocampi of 10 months old SPF, GF and $\mathrm{ABX}$ 5xFAD mice and non-transgenic controls (Fig. 6A \& B). In contrast to 4 months old animals, differences in microglial densities became indistinguishable in the $5 \mathrm{xFAD}$ mice at 10 months of age. In addition, the numbers of $\mathrm{TR}^{+} \mathrm{A} \beta$ plaque-associated microglia were similar in all three experimental groups (Fig. 6C). In ex vivo flow cytometric analysis, the percentage of methoxy-X-O4 ${ }^{+}$microglia showed no differences (Fig. 6D - F, Suppl. Fig. 5B), thus demonstrating the age-dependency of microbiota-controlled microglial $\mathrm{A} \beta$ phagocytosis.

\section{Discussion}

Recent studies uncovered an essential contribution of host microbiota during neurodegenerative diseases including $\mathrm{AD}$, however, the cellular and functional mechanisms involved in the disease progression are not yet understood. Here, we examined the influence of distinct gut microbiota modulation strategies on microglia in the context of AD. Colonized 5xFAD mice, housed under SPF conditions, showed robust hippocampal A $\beta$ pathology at early (4 months) and later disease stages (10 months), resulting thereby in disease stage-dependent neuronal loss and hippocampal-associated memory 


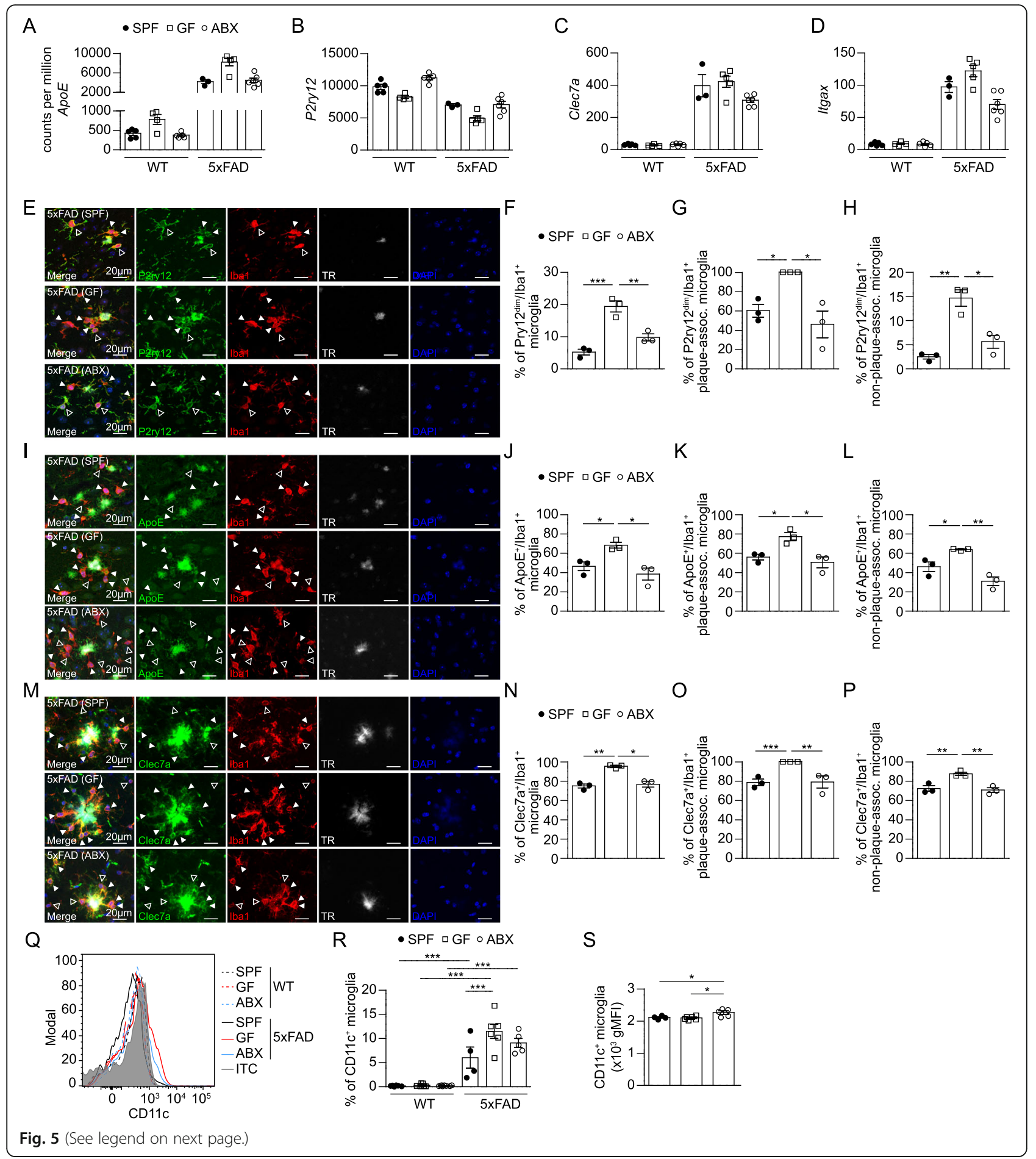


(See figure on previous page.)

Fig. 5 Altered expression of activation markers in hippocampal microglia from GF 5xFAD mice. Expression levels (counts per million) of (a) ApoE, (b) P2ry12, (c) Clec7a and (d) Itgax in hippocampal microglia from SPF, GF and ABX-treated 5xFAD and age-matched WT mice, based on RNA-seq data depicted in Fig. 4. Each symbol represents one mouse. Data are presented as mean \pm s.e.m. (e) Representative immunofluorescence images of $\mathrm{Iba}^{+}$(red), P2ry $12^{+}$(green) microglia and TR ${ }^{+}$(white) AB on parasagittal hippocampal sections from SPF, GF and ABX-treated 5xFAD mice. Nuclei were stained with DAPI (blue). Scale bar: $50 \mu \mathrm{m}$. White arrowheads indicate P2ry $12^{\mathrm{dim}} / \mathrm{lba} 1^{+}$microglia and non-filled arrowheads show P2ry $12^{\text {bright }} / \mathrm{lba} 1^{+}$microglia. Quantification of the percentage of $(\mathbf{f})$ total parenchymal P2ry $12^{\mathrm{dim}} / \mathrm{lba}^{+}$microglia $(\mathbf{g}) \mathrm{TR}^{+}$plaque-associated $\mathrm{P} 2 \mathrm{ry} 12^{\mathrm{dim}} / \mathrm{lba} 1^{+}$microglia and (h) non-plaque-associated P2ry $12^{\mathrm{dim}} / \mathrm{Iba} 1^{+}$microglia in hippocampi of SPF, GF and ABX-treated 5xFAD mice. (i) Representative immunofluorescence images of $\mathrm{Iba}^{+}$(red) $\mathrm{ApoE}^{+}$(green) microglia and $\mathrm{TR}^{+}$(white) A 3 on parasagittal hippocampal sections from SPF, GF and ABX-treated 5xFAD mice. Nuclei were stained with DAPI (blue). Scale bar: $50 \mu \mathrm{m}$. White arrowheads indicate ApoE $/ \mathrm{lba} 1^{+} \mathrm{microglia}$

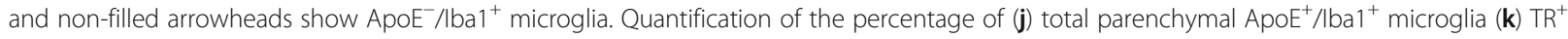
plaque-associated ApoE $\mathrm{Aba1}^{+}$microglia and (I) non-plaque-associated ApoE $\mathrm{Elba1}^{+}$microglia in hippocampus from SPF, GF and ABX-treated 5XFAD mice. (m) Representative immunofluorescence images of $\mathrm{Iba}^{+}{ }^{+}$(red) Clec7a ${ }^{+}$microglia (green) and $\mathrm{TR}^{+}$(white) on coronal hippocampal sections from SPF, GF and ABX-treated 5xFAD mice. Nuclei were stained with DAPI (blue). Scale bar: $50 \mu \mathrm{m}$. White arrowheads indicate Clec7a ${ }^{+} / \mathrm{lba1}{ }^{+}$ microglia and non-filled arrowheads show $\mathrm{Clec} 7 \mathrm{a}^{-} / \mathrm{lba} 1^{+}$microglia. Quantification of the percentage of (n) total parenchymal $\mathrm{Clec} 7 \mathrm{a}^{+} / \mathrm{lba} 1^{+} \mathrm{microglia}$ (o) $\mathrm{TR}^{+}$plaque-associated Clec $7 \mathrm{a}^{+} / \mathrm{Iba} 1^{+}$microglia and (p) non-plaque-associated Clec $7 \mathrm{a}^{+} / \mathrm{lba} 1^{+}$microglia in hippocampus of SPF, GF and ABX-treated 5xFAD mice. Each symbol represents one mouse. At least three slides were examined per individual mouse. Data are presented as mean \pm s.e.m. Significant differences were determined by one-way ANOVA ( $\left.{ }^{*} P<0.05,{ }^{*} \mathrm{P}<0.01,{ }^{* * *} \mathrm{P}<0.001\right)$. Data are representative of two independent experiments. (q) Representative cytometric graph of CD11c ${ }^{+}$labelled microglia from SPF (black line), GF (red line) and ABX-treated (blue line) 5xFAD mice and respective age-matched WT mice (dashed lines), compared to the isotype control (green line). In addition, quantifications of (r) percentages and (s) geometric mean fluorescence intensities (gMFI) of CD11 $\mathrm{c}^{+}$microglia cells are depicted. Each symbol represents one mouse. Data are presented as mean \pm s.e.m. Significant differences were determined by two-way ANOVA followed by Bonferroni's post-hoc comparison test or by one-way ANOVA followed by Tukey's post-hoc comparison test $\left({ }^{*} \mathrm{P}<0.05,{ }^{* * *} \mathrm{P}<0.001\right)$. Data are representative of three independent experiments

deficits. In contrast, constitutive (GF) or induced (ABX) depletion of the host's bacteria significantly alleviated $A \beta$ depositions and improved memory function. Importantly, constitutive and induced microbiota depletion strategies resulted in activation of different $A \beta$ clearing mechanisms by the brain's endogenous macrophages, the microglia. In GF 5xFAD mice microglial $A \beta$ uptake was increased, while this was not the case upon ABX-treatment of 5xFAD mice.

In general, commensal bacteria are a well-known factor in maintaining the host's physiology [41], including the innate and adaptive immune system in several tissues and compartments [16]. Moreover, alterations in the microbiota composition were linked to various human diseases, including several CNS disorders $[7,45,55$, $57,58]$. Their contribution to neurological health is studied extensively, however it is partially challenging to elucidate whether gut bacteria are a causative, propagative, or preventive factor during human health and disease. Nevertheless, growing evidence from preclinical studies suggests that gut bacteria are an essential factor contributing to CNS homeostasis and pathological conditions $[6,14,20,42,50]$. We previously identified that the commensal bacteria (presumably in the gut, due to their highest abundance) maintain microglial maturation and function in the CNS as evident by increased microglial numbers, highly branched arborization and altered gene expression patterns [12]. Interestingly, already during embryonic brain development, microglia features are essentially controlled by gut microbiota in a genderdependent manner [54].

In accordance with our own and previous observations $[18,34]$ showing that host microbiota boost $\mathrm{AD}$ pathology, emerging evidence implies that gut microbes influence the pathogenesis of various neurodegenerative CNS diseases [4, 13, 43]. However, it remained largely unclear how this effect might be mediated. Harach and colleagues reported, for instance, less $\mathrm{Iba}^{+}$microglia accumulation in AD 3.5 and 8 months old mice under GF conditions [18]. In contrast, by using the more aggressive $5 x F A D$ mouse model, we found in the hippocampus of GF housed transgenic mice an increased microglia density at 4 months of age. Of note, microglial numbers were equalized at later stages of the disease at 10 months of age. These data indicate certain limitations related to the different AD mouse models, as well as disease stagedependent divergences. Despite the usage of constitutive gnotobiotic mouse models, which are highly artificial and untranslatable to the human situation, long-term manipulation of the gut microbiota with a cocktail of antibiotics was described to result in decreased $A \beta$ burden in $\mathrm{APP}_{\mathrm{SWE}} / \mathrm{PS}_{\triangle \mathrm{E} 9} \mathrm{AD}$ model mice compared to non-treated controls [34]. In line, we observed diminished $A \beta$ depositions in ABX-treated 5xFAD mice at both, early (4 months) and late (10 months) time points. This finding suggests that the disease pathology can be alleviated even at a progressed state, which could be useful for possible late therapeutic interventions. Notably, in ABX-treated $5 \mathrm{xFAD}$ mice microglial density turned out to be on same levels as observed in SPF housed transgenic controls, complementary to unchanged microglia abundance during homeostatic conditions in non-transgenic WT mice as described before [12, 35, 54]. Importantly, we determined that diminished $\mathrm{A} \beta$ burden in GF and ABX-treated 5xFAD mice resulted at later stages in reduced neuronal loss and in turn attenuated hippocampus-associated memory loss. 


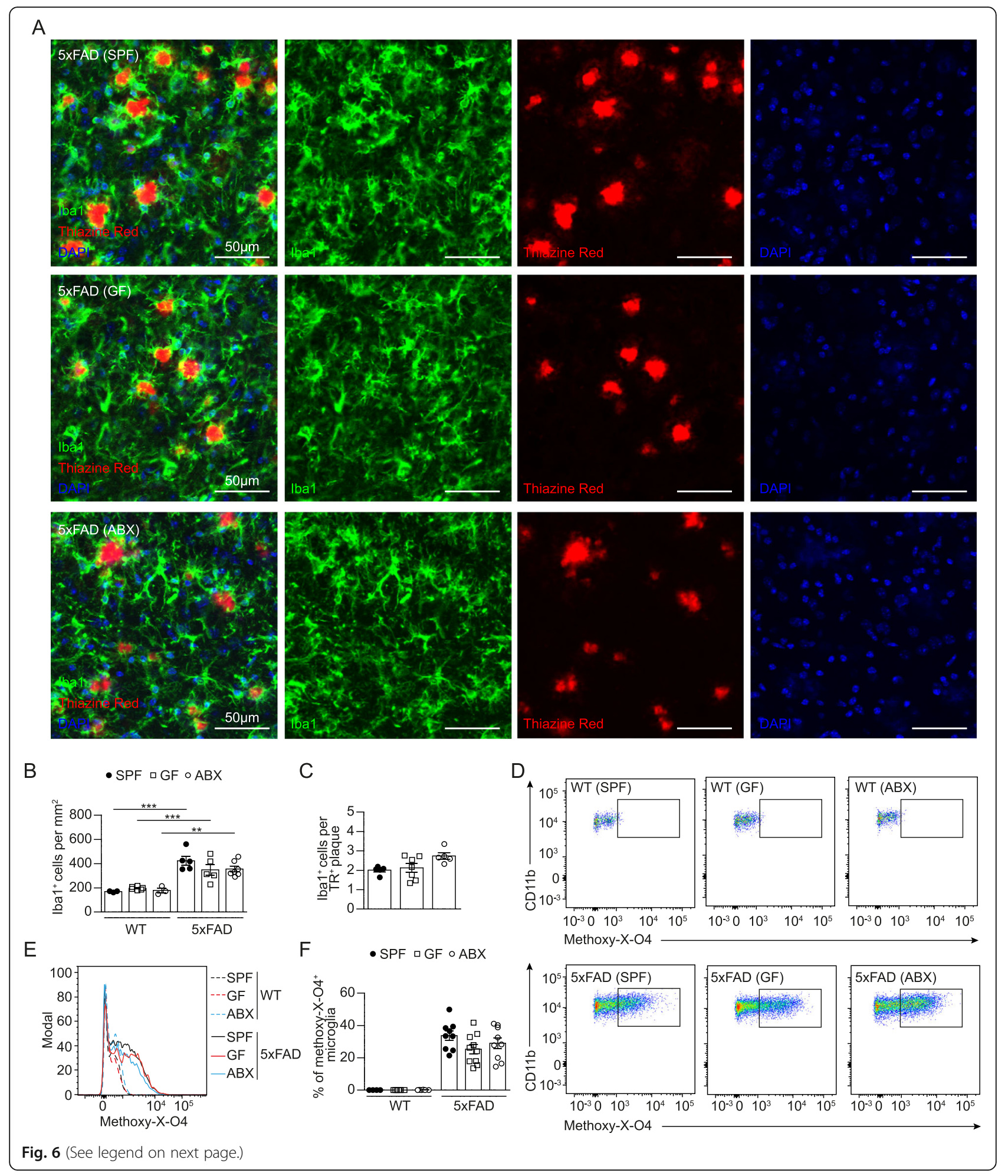


(See figure on previous page.)

Fig. 6 Equalized microglial A $\beta$ phagocytosis in the hippocampus of aged 5XFAD mice. (a) Representative immunofluorescence images of TR (red) and Iba1 (green) on coronal hippocampal sections from 10 months old 5xFAD mice. Nuclei were stained with DAPI (blue). Scale bar: $50 \mu m$. Quantification of (b) $\mid \mathrm{ba} 1^{+}$parenchymal in hippocampus of 5xFAD and age-matched WT mice. Quantification of (c) TR ${ }^{+}$plaque-associated microglia in 5xFAD mice. Each symbol represents one mouse. Data are presented as mean \pm s.e.m. Significant differences were determined by two-way ANOVA followed by Bonferroni post-hoc comparison test ( ${ }^{* * P}<0.01$, ${ }^{* *} \mathrm{P}<0.001$ ). (d) Gating of CD11 b ${ }^{+}$methoxy-XO4 ${ }^{+}$microglia from SPF, GF and ABX-treated 5xFAD mice and age-matched WT controls. Representative flow cytometric dot plots are shown. (e) Representative cytometric graph of methoxy-X-O4+ labelled microglia from SPF (black line), GF (red line) and ABX-treated (blue line) 5xFAD mice and respective WT controls (dashed lines). (f) Quantification of percentages of methoxy-X-O4+ labelled microglia cells are depicted. Each symbol represents one mouse. Data are presented as mean \pm s.e.m.: No significant differences were detected by two-way ANOVA followed by Bonferroni post-hoc comparison test. Data are representative of three independent experiments

How does the microbiota shape AD progression? Generally, the equilibrium between $A \beta$ production and clearance is regarded to be crucial for the $\mathrm{A} \beta$ burden in $\mathrm{AD}$ brain [22]. In addition, impaired $A \beta$ removal rather than increased $A \beta$ generation has been associated to the etiology of sporadic AD in humans [32]. The production of $A \beta$ and APP processing was not altered in transgenic SPF, GF and ABX-treated 5xFAD mice. However, GF 5xFAD mice displayed an increased microglia accumulation close to $A \beta$ plaques, containing more $A \beta$ debris intracellularly compared to 4 months old SPF and ABX-treated 5xFAD mice, indicative of amplified phagocytic removal of $A \beta$. Consistent with these observations, several molecules regulating phagocytosis, such as Trem2, its adapter TYRO protein tyrosine kinase binding protein (Tyrobp), complement receptors (e.g. complement $\mathrm{C} 1 \mathrm{q}$ subcomponent subunit B, C1qb) and Apoe were elevated in microglia from GF $5 x F A D$ mice. For the lipoproteins, and in particular APOE, it is known that compact $A \beta$ aggregates can be phagocytosed more efficiently by microglia $[52,61]$. Remarkably, several genes (such as Clec7a, P2ry12, Itgax and others), that have been recently associated to $A \beta$ activated microglia in AD mouse models [23, 27], as well as in human AD brains [56, 63], displayed altered expression in microglia from GF 5xFAD mice, indicating a certain microbiota-dependent influence on this microglial activation state. In addition, the microglial expression levels of genes attributed to neuroinflammation and chemokine signaling pathways were suppressed under GF conditions and partially reduced after $A B X$ treatment. Despite the potential direct neurotoxic effects of such molecules, as it was proposed in the context of Parkinson's diseases [43], cytokine expression is also implicated in affecting microglial phagocytosis [3]. Of note, the observed elevated uptake of $\mathrm{A} \beta$ depositions in GF 5xFAD mice was found to be equalized at later stages of the disease. In contrast to GF $5 x F A D$ mice, acutely induced microbiota depletion by $A B X$ did not increase microglial uptake of $A \beta$ at early or late stages of the disease as compared to the SPF condition, suggesting other mechanisms of microbiota-driven A $\beta$ clearance.

It was further reported that conventionally housed transgenic APPPS1 mice harbor alterations in gut microbiota composition, including increased abundance of e.g. Rikenellaceae, compared to the WT littermates. In the 5xFAD mouse model, slight changes in the composition of the fecal microbiota were also described compromising Bacteroidetes and Firmicutes [5]. However, in our $16 \mathrm{~S}$ analysis of caecal contents we observed no major alterations (with a q-value $<0.05$ ). In a rather small study, microbiota analysis of AD patients revealed declined microbial diversity as compared with control patients [58]. However, clinical implications of microbiota alterations need to be further clarified in future studies.

In conclusion, we found that host microbiota controlled microglia-mediated uptake of $A \beta$ depositions in the $5 \mathrm{xFAD}$ mouse model of $\mathrm{AD}$, whereas we observed different effects of constitutive (as in GF conditions) and induced microbiota modulation (by $\mathrm{ABX}$ ) as well as disease stage-dependent mechanisms. RNA-seq analysis of FACS-purified hippocampal microglia uncovered distinct microbiota-dependent gene expression patterns including genes attributed to e.g. phagocytosis or complement signaling. Further, we found genes ascribed to AD-linked activation of microglia such as Cst7, Clec7a, Apoe or Itgax being expressed in a microbiota-dependent manner. These results provide deeper knowledge about the high plasticity of the gut-microglia connection and treatment of microglia-mediated CNS diseases.

\section{Supplementary information}

Supplementary information accompanies this paper at https://doi.org/10. 1186/s40478-020-00988-5.

\footnotetext{
Additional file 1: Supplementary Fig. 1: Determination of intestinal bacterial loads and microbiota composition analysis. (A) Photograph of caeca from 4- and 10- months old SPF, GF and ABX-treated mice with ruler for scaling and (B) relative caecal weight referred to the body weight, and $(\mathbf{C})$ absolute body weight. Each symbol represents one mouse. Data are presented as mean \pm s.e.m. Significant differences were determined by one-way ANOVA followed by Tukey's post-hoc comparison test ( $\left.{ }^{* *} P<0.001\right)$. Data are representative of four independent experiments. (D) Gating on DAPI-Syto9 ${ }^{+}$live bacteria of fecal samples from SPF, GF and ABX-treated mice. Representative flow cytometric dot plots are shown. (E) Percentages of live gram + and gram- bacteria and (F) quantification of live bacteria per mg fecal sample are depicted. Each symbol represents one mouse. Data are presented as mean \pm s.e.m. Significant differences were determined by one-way ANOVA followed by Tukey's post-hoc comparison test (***P $<0.001$ ). Data are representative
} 
of four independent experiments. (G-J) Microbial species richness (alpha diversity; Shannon and Simpson indices) in caecal contents from 4 months old SPF and ABX-treated 5XFAD mice and respective agematched WT controls. (K) Microbial clustering is shown based on BrayCurtis dissimilarity principal coordinate analysis ( $\mathrm{PCOA}$ ) metrics of caecal contents from 4 months old SPF and ABX-treated 5XFAD mice and respective age-matched WT controls. Ellipsoids represent a 95\% confidence interval surrounding each group. Non-parametric analysis of variance (Adonis) was used to test significant difference between groups on PCoA plot; $p<0.001$ for tested groups.

Additional file 2: Supplementary Fig. 2. Absence of host microbiota reduces hippocampal A $\beta$ depositions in 5XFAD mice. (A) Representative immunofluorescence images of $6 \mathrm{E} 10^{+}$(red) compact and diffuse $A \beta$ plaques in the hippocampus of 4 months old SPF, GF and ABX-treated 5 XFAD mice. Nuclei were stained with DAPI (blue). Overview of hippocampus and magnification of subiculum (dashed line) are shown. Scale bars represent $300 \mu \mathrm{m}$ (overview) and $50 \mu \mathrm{m}$ (insert). (B) Quantification of the number of $6 \mathrm{E} 10^{+} \mathrm{A} \beta$-plaques per $\mathrm{mm}^{2}$, (C) percentage of $6 \mathrm{E} 10^{+}$area and $(\mathbf{D})$ average $6 \mathrm{E} 10^{+}$plaque size $\left(\mu \mathrm{m}^{2}\right)$ in coronal hippocampal sections of SPF, GF and ABX-treated 5xFAD mice. Each symbol represents one mouse. Data are presented as mean \pm s.e.m. Significant differences were determined by one-way ANOVA followed by Tukey's post-hoc comparison test ( $\left.{ }^{* *} P<0.001\right)$. Data are representative of four independent experiments. (E) Insoluble $A \beta 42 / A \beta 40$ and $(\mathbf{F})$ soluble $A \beta 42 / A \beta 40$ ratio of hippocampal brain extracts from 4 months old SPF, GF and ABX-treated 5xFAD mice. ELISA for $(\mathbf{G})$ insoluble A 342 , (H) insoluble $A \beta 40$, (I) soluble $A \beta 42$, (J) soluble $A \beta 40,(\mathbf{K})$ ratio of insoluble $A \beta 42 / A \beta 40$ and ratio of soluble $A \beta 42 / A \beta 40$ of hippocampal brain extracts of 10 months old SPF, GF and ABX-treated 5XFAD mice. Each symbol represents one mouse. Data are presented as mean \pm s.e.m. Significant differences were determined by one-way ANOVA followed by Tukey's post-hoc comparison test ( ${ }^{* *} P<$ $0.01,{ }^{* * *} P<0.001$ ). Data are representative of two independent experiments. (L) Representative immunoblots of hippocampal brain homogenates of 10 months old SPF, GF and ABX-treated $5 \times$ FAD mice against human APP-FL, CTF- $\beta$, CTF- $\alpha$, BACE1, ADAM10, PS1, PS2, PEN2, Nicastrin, and $A \beta$ (6E10). $\beta$-Actin was used as loading control. Each lane represents one mouse. Quantification of (M) APP-FL, (N), CTF- $\beta,(\mathbf{O})$ CTF- $\alpha,(\mathbf{P})$ BACE1, (Q) ADAM10, (R) PS1, (S) PS2, (T) PEN2, (U) Nicastrin, and (V) AB (6E10) protein levels normalized to $\beta$-Actin. Each symbol represents one mouse. Data are presented as mean \pm s.e.m. Significant differences were determined by one-way ANOVA followed by Tukey's post-hoc comparison test $\left({ }^{*} P<0.05\right.$, $\left.{ }^{* *} P<0.01\right)$. Homogenates of three mice per group were used. (W) Representative immunofluorescence images of $6 \mathrm{E} 10^{+}$compact and diffuse $A \beta$ plaques in the hippocampus of 10 months old $5 \times F A D$ mice. Nuclei were stained with DAPI (blue). Overview of hippocampus and magnification of subiculum (dashed line) are shown. Scale bars represent $300 \mu \mathrm{m}$ (overview) and $50 \mu \mathrm{m}$ (insert). (X) Quantification of the number of $6 \mathrm{E} 10^{+} \mathrm{A} \beta$-plaques per area $\left(\mathrm{mm}^{2}\right),(\mathbf{Y})$ percentage of $6 \mathrm{E} 10^{+}$area and $(\mathbf{Z})$ average $6 \mathrm{E} 10^{+}$plaque size $\left(\mu \mathrm{m}^{2}\right)$. Each symbol represents one mouse. Data are presented as mean \pm s.e.m. Significant differences were determined by one-way ANOVA followed by Tukey's post-hoc comparison test $\left({ }^{*} \mathrm{P}<0.05,{ }^{* *} P<0.001\right)$. Data are representative of three independent experiments.

Additional file 3: Supplementary Fig. 3. Memory function in 4 months old SPF, GF and ABX-treated 5xFAD mice. (A) Ratio of right versus left arm entries in the T-maze spontaneous alternation test of 10 months old SPF and GF or (B) SPF and ABX-treated 5XFAD and agematched WT mice. (C-F) T-maze of 4 months old SPF and GF mice or (G-J) SPF and ABX-treated 5xFAD, as well as respective age-matched WT mice. (K-M) Novel object recognition task of 4 months old SPF and GF 5XFAD, as well as age-matched WT mice or (N-P) SPF and ABX-treated 5XFAD, as well as WT mice. Each symbol represents one mouse. Data are presented as mean \pm s.e.m. Significant differences were determined by two-way ANOVA followed by Bonferroni's post-hoc comparison test $\left({ }^{*} P<0.05,{ }^{* *} P<0.01,{ }^{* *} P<0.001\right)$. Data are representative of three independent experiments. (Q) Representative immunofluorescence images of $\mathrm{NeuN}^{+}$neurons (green) and $\mathrm{TR}^{+}$(red) compact Aß-plaques in the subiculum of the hippocampus of 4 months old SPF, GF and ABX-treated 5xFAD and respective age-matched WT mice. Nuclei were stained with DAPI (blue). Overview of hippocampus and magnification of subiculum (dashed line) are shown. Scale bars represent $200 \mu \mathrm{m}$ (overview) and $50 \mu \mathrm{m}$ (insert). (R) Quantification of the number of $\mathrm{NeuN}^{+}$neurons per $\mathrm{mm}^{2}$ in the subiculum (Sub) of sagittal hippocampal sections from SPF, GF and ABX-treated 5xFAD and WT mice. Each symbol represents one mouse. Data are presented as mean \pm s.e.m. No significant differences were determined by two-way ANOVA followed by Bonferroni's post-hoc comparison test. Data are representative of two independent experiments.

Additional file 4: Supplementary Fig. 4. Microglial density in hippocampus of 4 months old 5xFAD mice. (A) Representative immunofluorescence images of $\mid \mathrm{bal}^{+}$(green) microglia on coronal hippocampal sections of 4 months old SPF, GF and ABX-treated WT mice. Nuclei were stained with DAPI (blue). Scale bar: 50 mm. (B) Representative immunofluorescence images of 6E10 (red) and Iba1 (green) on coronal hippocampal sections of 4 months old 5XFAD mice. Nuclei were stained with DAPI (blue). Scale bar: $50 \mu \mathrm{m}$. (C) Quantification of 6E10 $0^{+}$plaqueassociated microglia. Each symbol represents one mouse. Data are represented as means \pm s.e.m. Significant differences were determined by one-way ANOVA followed by Tukey's post-hoc comparison test ${ }^{* *} * 0.01$, $\left.{ }^{* * *} \mathrm{P}<0.001\right)$. Data are representative of two independent experiments.

Additional file 5: Supplementary Fig. 5. Gating of hippocampal microglia in 5XFAD mice and non-transgenic controls. Gating of CD11 $\mathrm{b}^{+} \mathrm{CD} 45^{\text {low }}$ microglia from (A) 4 months old or (B) 10 months old SPF, GF and ABX-treated 5xFAD mice and age-matched WT controls. Representative dot plots are shown.

Additional file 6. Table 1. Maaslin Analysis Output (H2O Group for Genotype Comparison).

Additional file 7. Table 2. Maaslin Analysis Output (ABX Groups for Genotype Comparison).

Additional file 8. Table 3. Venn-diagram related genes.

Additional file 9. Table 4. List of DEG

Additional file 10. Table 5. Pathway analysis SPF FAD vs WT.

Additional file 11. Table 6. Pathway analysis GF FAD vs WT.

Additional file 12. Table 7. Pathway analysis ABX FAD vs WT.

\section{Acknowledgments}

We thank Eileen Barleon, Tina el Gaz, Katrin Seidel, Anna Lena Hrabě de Angelis and Janika Sosat for excellent technical assistance. Kathleen McCoy introduced the GF 5xFAD line at CMF (Bern, Switzerland). Jakob Zimmermann established flow cytometric analysis of microbial load in fecal samples. We are grateful to J. Bodinek-Wersing for cell sorting. RNAsequencing was performed at KFB, Center of Excellence for Fluorescent Bioanalytics, Regensburg. MP is supported by the Sobek Foundation, the ErnstJung Foundation, the DFG (SFB 992, SFB1160, SFB/TRR167, Reinhart Koselleck Grant, Gottfried Wilhelm Leibniz-Prize), the Ministry of Science, Research and Arts, Baden-Wuerttemberg (Sonderlinie 'Neuroinflammation'). TB is supported by by the Dr. Kübler-Chicken-Stiftung (1027095701). DE is supported by the DFG (SFB/TRR167) and the Berta-Ottenstein-Programme for Clinician Scientists, Faculty of Medicine, University of Freiburg.

\section{Authors' contributions}

$\mathrm{CM}$ conducted the majority of the experiments and made the figures. $\mathrm{DE}$ wrote the manuscript and $\mathrm{CM}, \mathrm{ND}, \mathrm{OM}$ and $\mathrm{MP}$ edited the manuscript. $\mathrm{DE}$, $J N, N D, O M, D S, M M L, P S$ and TB provided help with the tissue processing and experiments. OS analyzed the RNA-sequencing data. MGdeA, SCGV and AJM bred GF animals. MP and DE supervised the project. The author(s) read and approved the final manuscript.

\section{Availability of data and materials}

All sequencing data (RNA-seq) are available at Gene Expression Omnibus (GEO: GSE154428).

\section{Competing interests}

The authors declare no competing interests. 


\section{Author details}

Institute of Neuropathology, University of Freiburg, Breisacher Str. 64, 79106 Freiburg, Germany. ${ }^{2}$ Faculty of Biology, University of Freiburg, Freiburg, Germany. ${ }^{3}$ Maurice E. Müller Laboratories, Department for Biomedical Research (DBMR), University Clinic of Visceral Surgery and Medicine, Inselspital, University of Bern, Bern, Switzerland. ${ }^{4}$ Institute of Virology, Medical Center University of Freiburg, Freiburg, Germany. ${ }^{5}$ Department of Neurology, Medical Center University of Freiburg, Freiburg, Germany. ${ }^{6} \mathrm{Center}$ for Basics in NeuroModulation (NeuroModulBasics), Faculty of Medicine, University of Freiburg, Freiburg, Germany. ${ }^{7}$ Signalling Research Centres BIOSS and CIBSS, University of Freiburg, Freiburg, Germany. ${ }^{8}$ Berta-Ottenstein-Programme, Faculty of Medicine, University of Freiburg, Freiburg, Germany.

Received: 3 July 2020 Accepted: 4 July 2020

Published online: 29 July 2020

\section{References}

1. Andrews S (2019) FastQC: a quality control tool for high throughput sequence data. http://www.bioinformatics.babraham.ac.uk/projects/fastqc

2. Antunes M, Biala G (2012) The novel object recognition memory: neurobiology, test procedure, and its modifications. Cogn Process 13:93110. https://doi.org/10.1007/s10339-011-0430-z

3. Babcock AA, Ilkjaer $L$, Clausen BH, Villadsen B, Dissing-Olesen $L$, Bendixen AT, Lyck L, Lambertsen KL, Finsen B (2015) Cytokine-producing microglia have an altered beta-amyloid load in aged APP/PS1 Tg mice. Brain Behav Immun 48:86-101. https://doi.org/10.1016/j.bbi.2015.03.006

4. Blacher E, Bashiardes S, Shapiro H, Rothschild D, Mor U, Dori-Bachash M, Kleimeyer C, Moresi C, Harnik Y, Zur Met al (2019) Potential roles of gut microbiome and metabolites in modulating ALS in mice. Nature https://doi. org/10.1038/s41586-019-1443-5

5. Brandscheid C, Schuck F, Reinhardt S, Schafer KH, Pietrzik CU, Grimm M, Hartmann T, Schwiertz A, Endres K (2017) Altered gut microbiome composition and Tryptic activity of the 5xFAD Alzheimer's mouse model. J Alzheimers Dis 56:775-788. https://doi.org/10.3233/JAD-160926

6. Bravo JA, Forsythe $P$, Chew MV, Escaravage E, Savignac HM, Dinan TG, Bienenstock J, Cryan JF (2011) Ingestion of Lactobacillus strain regulates emotional behavior and central GABA receptor expression in a mouse via the vagus nerve. Proc Natl Acad Sci U S A 108:16050-16055. https://doi.org/ 10.1073/pnas.1102999108

7. Brenner D, Hiergeist A, Adis C, Mayer B, Gessner A, Ludolph AC, Weishaupt $\mathrm{JH}$ (2018) The fecal microbiome of ALS patients. Neurobiol Aging 61:132137. https://doi.org/10.1016/..neurobiolaging.2017.09.023

8. Caporaso JG, Kuczynski J, Stombaugh J, Bittinger K, Bushman FD, Costello EK, Fierer N, Pena AG, Goodrich JK, Gordon Jl et al (2010) QIIME allows analysis of high-throughput community sequencing data. Nat Methods 7 : 335-336. https://doi.org/10.1038/nmeth.f.303

9. Collaborators GBDD (2019) Global, regional, and national burden of Alzheimer's disease and other dementias, 1990-2016: a systematic analysis for the global burden of disease study 2016. Lancet Neurol 18:88-106. https://doi.org/10.1016/S1474-4422(18)30403-4

10. Datta M, Staszewski $O$, Raschi E, Frosch M, Hagemeyer N, Tay TL, Blank T, Kreutzfeldt M, Merkler D, Ziegler-Waldkirch S et al (2018) Histone Deacetylases 1 and 2 regulate microglia function during development, homeostasis, and Neurodegeneration in a context-dependent manner Immunity 48:514-529 e516. https://doi.org/10.1016/j.immuni.2018.02.016

11. Dobin A, Davis CA, Schlesinger F, Drenkow J, Zaleski C, Jha S, Batut P, Chaisson M, Gingeras TR (2013) STAR: ultrafast universal RNA-seq aligner Bioinformatics 29:15-21. https://doi.org/10.1093/bioinformatics/bts635

12. Erny D, Hrabe de Angelis AL, Jaitin D, Wieghofer $P$, Staszewski O, David E, Keren-Shaul H, Mahlakoiv T, Jakobshagen K, Buch T et al (2015) Host microbiota constantly control maturation and function of microglia in the CNS. Nat Neurosci 18:965-977. https://doi.org/10.1038/nn.4030

13. Erny D, Prinz M (2017) Microbiology: gut microbes augment neurodegeneration. Nature 544:304-305. https://doi.org/10.1038/ nature21910

14. Erny D, Prinz M (2020) How microbiota shape microglial phenotypes and epigenetics. Glia 68:1655-1672. https://doi.org/10.1002/glia.23822

15. Frankish A, Diekhans M, Ferreira AM, Johnson R, Jungreis I, Loveland J, Mudge JM, Sisu C, Wright J, Armstrong J et al (2019) GENCODE reference annotation for the human and mouse genomes. Nucleic Acids Res 47: D766-D773. https://doi.org/10.1093/nar/gky955
16. Geuking MB, Koller Y, Rupp S, McCoy KD (2014) The interplay between the gut microbiota and the immune system. Gut Microbes 5:411-418. https:// doi.org/10.4161/gmic.29330

17. Ginhoux F, Greter M, Leboeuf M, Nandi S, See P, Gokhan S, Mehler MF, Conway SJ, Ng LG, Stanley ER et al (2010) Fate mapping analysis reveals that adult microglia derive from primitive macrophages. Science 330:841845. https://doi.org/10.1126/science.1194637

18. Harach T, Marungruang N, Duthilleul N, Cheatham V, Mc Coy KD, Frisoni G, Neher JJ, Fak F, Jucker M, Lasser T et al (2017) Reduction of Abeta amyloid pathology in APPPS1 transgenic mice in the absence of gut microbiota. Sci Rep 7:41802. https://doi.org/10.1038/srep41802

19. Heberle H, Meirelles GV, da Silva FR, Telles GP, Minghim R (2015) InteractiVenn: a web-based tool for the analysis of sets through Venn diagrams. BMC Bioinformatics 16:169. https://doi.org/10.1186/s12859-015-0611-3

20. Hegstrand LR, Hine RJ (1986) Variations of brain histamine levels in germfree and nephrectomized rats. Neurochem Res 11:185-191. https://doi.org/ 10.1007/bf00967967

21. Hooper LV, Gordon JI (2001) Commensal host-bacterial relationships in the gut. Science 292:1115-1118

22. Hyman BT, Marzloff K, Arriagada PV (1993) The lack of accumulation of senile plaques or amyloid burden in Alzheimer's disease suggests a dynamic balance between amyloid deposition and resolution. J Neuropathol Exp Neurol 52:594-600. https://doi.org/10.1097/00005072199311000-00006

23. Keren-Shaul H, Spinrad A, Weiner A, Matcovitch-Natan O, Dvir-Szternfeld R, Ulland TK, David E, Baruch K, Lara-Astaiso D, Toth B et al (2017) A unique microglia type associated with restricting development of Alzheimer's disease. Cell 169(1276-1290):e1217. https://doi.org/10.1016/j. cell.2017.05.018

24. Kierdorf K, Erny D, Goldmann T, Sander V, Schulz C, Perdiguero EG, Wieghofer P, Heinrich A, Riemke P, Holscher C et al (2013) Microglia emerge from erythromyeloid precursors via Pu.1- and Irf8-dependent pathways. Nat Neurosci 16:273-280. https://doi.org/10.1038/nn.3318

25. Kierdorf K, Prinz M (2017) Microglia in steady state. J Clin Invest 127:32013209. https://doi.org/10.1172/JC190602

26. Kong L, Ge BX (2008) MyD88-independent activation of a novel actinCdc42/Rac pathway is required for toll-like receptor-stimulated phagocytosis. Cell Res 18:745-755. https://doi.org/10.1038/cr.2008.65

27. Krasemann S, Madore C, Cialic R, Baufeld C, Calcagno N, El Fatimy R, Beckers L, O'Loughlin E, Xu Y, Fanek $Z$ et al (2017) The TREM2-APOE pathway drives the transcriptional phenotype of dysfunctional microglia in neurodegenerative diseases. Immunity 47:566-581 e569. https://doi.org/10. 1016/j.immuni.2017.08.008

28. Lambert JC, Ibrahim-Verbaas CA, Harold D, Naj AC, Sims R, Bellenguez C, DeStafano AL, Bis JC, Beecham GW, Grenier-Boley B et al (2013) Metaanalysis of 74,046 individuals identifies 11 new susceptibility loci for Alzheimer's disease. Nat Genet 45: 1452-1458 Doi https://doi.org/10.1038/ ng.2802

29. Law CW, Chen Y, Shi W, Smyth GK (2014) Voom: precision weights unlock linear model analysis tools for RNA-seq read counts. Genome Biol 15:R29. https://doi.org/10.1186/gb-2014-15-2-r29

30. Liao Y, Smyth GK, Shi W (2014) featureCounts: an efficient general purpose program for assigning sequence reads to genomic features. Bioinformatics 30:923-930. https://doi.org/10.1093/bioinformatics/btt656

31. Liu R, Holik AZ, Su S, Jansz N, Chen K, Leong HS, Blewitt ME, Asselin-Labat ML, Smyth GK, Ritchie ME (2015) Why weight? Modelling sample and observational level variability improves power in RNA-seq analyses. Nucleic Acids Res 43:e97. https://doi.org/10.1093/nar/gkv412

32. Mawuenyega KG, Sigurdson W, Ovod V, Munsell L, Kasten T, Morris JC, Yarasheski KE, Bateman RJ (2010) Decreased clearance of CNS beta-amyloid in Alzheimer's disease. Science 330:1774. https://doi.org/10.1126/science.1197623

33. McMurdie PJ, Holmes S (2013) Phyloseq: an R package for reproducible interactive analysis and graphics of microbiome census data. PLoS One 8: e61217. https://doi.org/10.1371/journal.pone.0061217

34. Minter MR, Zhang C, Leone V, Ringus DL, Zhang X, Oyler-Castrillo P, Musch MW, Liao F, Ward JF, Holtzman DM et al (2016) Antibiotic-induced perturbations in gut microbial diversity influences neuro-inflammation and amyloidosis in a murine model of Alzheimer's disease. Sci Rep 6:30028. https://doi.org/10.1038/srep30028

35. Mohle L, Mattei $D$, Heimesaat MM, Bereswill $S$, Fischer A, Alutis M, French $T$, Hambardzumyan D, Matzinger P, Dunay IR et al (2016) Ly6C(hi) monocytes 
provide a link between antibiotic-induced changes in gut microbiota and adult hippocampal neurogenesis. Cell Rep 15:1945-1956. https://doi.org/10. 1016/j.celrep.2016.04.074

36. Morgan XC, Tickle TL, Sokol H, Gevers D, Devaney KL, Ward DV, Reyes JA, Shah SA, LeLeiko N, Snapper SB et al (2012) Dysfunction of the intestinal microbiome in inflammatory bowel disease and treatment. Genome Biol 13: R79. https://doi.org/10.1186/gb-2012-13-9-r79

37. Mathew NR, Vinnakota JM, Apostolova P, Erny D, Hamarsheh S, Andrieux G, Kim JS, Hanke K, Goldmann T, Chappell-Maor L et al (2019) Graft-versus-host disease of the CNS is mediated by TNF upregulation in microglia. J Clin Invest. https://doi.org/10.1172/JCl130272

38. Oakley H, Cole SL, Logan S, Maus E, Shao P, Craft J, Guillozet-Bongaarts A, Ohno M, Disterhoft J, Van Eldik L et al (2006) Intraneuronal beta-amyloid aggregates, neurodegeneration, and neuron loss in transgenic mice with five familial Alzheimer's disease mutations: potential factors in amyloid plaque formation. J Neurosci 26:10129-10140. https://doi.org/10.1523/ JNEUROSCI.1202-06.2006

39. Prinz M, Jung S, Priller J (2019) Microglia biology: one century of evolving concepts. Cell 179:292-311. https://doi.org/10.1016/j.cell.2019.08.053

40. Raivo K (2019) Pheatmap: pretty Heatmaps. R package version 1.0.12., City

41. Rowland I, Gibson G, Heinken A, Scott K, Swann J, Thiele I, Tuohy K (2018) Gut microbiota functions: metabolism of nutrients and other food components. Eur J Nutr 57:1-24. https://doi.org/10.1007/s00394017-1445-8

42. Sadler R, Cramer JV, Heindl S, Kostidis S, Betz D, Zuurbier KR, Northoff BH, Heijink M, Goldberg MP, Plautz EJ et al (2020) Short-chain fatty acids improve Poststroke recovery via immunological mechanisms. J Neurosci 40: 1162-1173. https://doi.org/10.1523/JNEUROSCl.1359-19.2019

43. Sampson TR, Debelius JW, Thron T, Janssen S, Shastri GG, Ilhan ZE, Challis C, Schretter CE, Rocha S, Gradinaru V et al (2016) Gut microbiota regulate motor deficits and Neuroinflammation in a model of Parkinson's disease. Cell 167(1469-1480):e1412. https://doi.org/10.1016/j. cell.2016.11.018

44. Sarlus H, Heneka MT (2017) Microglia in Alzheimer's disease. J Clin Invest 127:3240-3249. https://doi.org/10.1172/JC190606

45. Scheperjans F, Aho V, Pereira PA, Koskinen K, Paulin L, Pekkonen E, Haapaniemi E, Kaakkola S, Eerola-Rautio J, Pohja M et al (2015) Gut microbiota are related to Parkinson's disease and clinical phenotype. Mov Disord 30:350-358. https://doi.org/10.1002/mds.26069

46. Schulz C, Gomez Perdiguero E, Chorro L, Szabo-Rogers H, Cagnard N, Kierdorf K, Prinz M, Wu B, Jacobsen SE, Pollard JW et al (2012) A lineage of myeloid cells independent of Myb and hematopoietic stem cells. Science 336:86-90. https://doi.org/10.1126/science.1219179

47. Selkoe DJ (2012) Preventing Alzheimer's disease. Science 337:1488-1492. https://doi.org/10.1126/science.1228541

48. Sperling R, Mormino E, Johnson K (2014) The evolution of preclinical Alzheimer's disease: implications for prevention trials. Neuron 84:608-622. https://doi.org/10.1016/j.neuron.2014.10.038

49. Spowart-Manning L, van der Staay FJ (2004) The T-maze continuous alternation task for assessing the effects of putative cognition enhancers in the mouse. Behav Brain Res 151:37-46. https://doi.org/10.1016/j.bbr.2003.08.004

50. Sudo N, Chida Y, Aiba Y, Sonoda J, Oyama N, Yu XN, Kubo C, Koga Y (2004) Postnatal microbial colonization programs the hypothalamic-pituitaryadrenal system for stress response in mice. J Physiol 558:263-275. https:// doi.org/10.1113/jphysiol.2004.063388

51. Tanzi RE (2012) The genetics of Alzheimer disease. Cold Spring Harb Perspect Med 2. https://doi.org/10.1101/cshperspect.a006296

52. Terwel D, Steffensen KR, Verghese PB, Kummer MP, Gustafsson JA, Holtzman DM, Heneka MT (2011) Critical role of astroglial apolipoprotein $E$ and liver $X$ receptor-alpha expression for microglial Abeta phagocytosis. J Neurosci 31: 7049-7059. https://doi.org/10.1523/JNEUROSCI.6546-10.2011

53. Theriault P, ElAli A, Rivest S (2015) The dynamics of monocytes and microglia in Alzheimer's disease. Alzheimers Res Ther 7:41. https://doi.org/ 10.1186/s13195-015-0125-2

54. Thion MS, Low D, Silvin A, Chen J, Grisel P, Schulte-Schrepping J, Blecher R, Ulas T, Squarzoni P. Hoeffel G et al (2018) Microbiome influences prenatal and adult microglia in a sex-specific manner. Cell 172(500-516):e516. https://doi.org/10.1016/j.cell.2017.11.042

55. Tremlett H, Bauer KC, Appel-Cresswell S, Finlay BB, Waubant E (2017) The gut microbiome in human neurological disease: a review. Ann Neurol 81: 369-382. https://doi.org/10.1002/ana.24901
56. Uchihara T, Duyckaerts C, He Y, Kobayashi K, Seilhean D, Amouyel P, Hauw JJ (1995) ApoE immunoreactivity and microglial cells in Alzheimer's disease brain. Neurosci Lett 195:5-8. https://doi.org/10.1016/0304-3940(95)11763-m

57. Unger MM, Spiegel J, Dillmann KU, Grundmann D, Philippeit H, Burmann J, Fassbender K, Schwiertz A, Schafer KH (2016) Short chain fatty acids and gut microbiota differ between patients with Parkinson's disease and agematched controls. Parkinsonism Relat Disord 32:66-72. https://doi.org/10. 1016/j.parkreldis.2016.08.019

58. Vogt NM, Kerby RL, Dill-McFarland KA, Harding SJ, Merluzzi AP, Johnson SC, Carlsson CM, Asthana S, Zetterberg H, Blennow K et al (2017) Gut microbiome alterations in Alzheimer's disease. Sci Rep 7:13537. https://doi.org/10.1038/s41598-017-13601-y

59. Wang Y, Cella M, Mallinson K, Ulrich JD, Young KL, Robinette ML, Gilfillan S, Krishnan GM, Sudhakar S, Zinselmeyer BH et al (2015) TREM2 lipid sensing sustains the microglial response in an Alzheimer's disease model. Cell 160: 1061-1071. https://doi.org/10.1016/j.cell.2015.01.049

60. Wang Y, Ulland TK, Ulrich JD, Song W, Tzaferis JA, Hole JT, Yuan P, Mahan TE, Shi Y, Gilfillan S et al (2016) TREM2-mediated early microglial response limits diffusion and toxicity of amyloid plaques. J Exp Med 213:667-675. https://doi.org/10.1084/jem.20151948

61. Yeh FL, Wang Y, Tom I, Gonzalez LC, Sheng M (2016) TREM2 binds to Apolipoproteins, including APOE and CLU/APOJ, and thereby facilitates uptake of amyloid-Beta by microglia. Neuron 91:328-340. https://doi.org/10. 1016/j.neuron.2016.06.015

62. Yilmaz B, Juillerat $P$, Oyas $O$, Ramon C, Bravo FD, Franc $Y$, Fournier $N$, Michetti P, Mueller C, Geuking M et al (2019) Microbial network disturbances in relapsing refractory Crohn's disease. Nat Med 25:323-336. https://doi.org/10.1038/s41591-018-0308-z

63. Yin Z, Raj D, Saiepour N, Van Dam D, Brouwer N, Holtman IR, Eggen BJL, Moller T, Tamm JA, Abdourahman A et al (2017) Immune hyperreactivity of Abeta plaque-associated microglia in Alzheimer's disease. Neurobiol Aging 55:115-122. https://doi.org/10.1016/j.neurobiolaging.2017.03.021

64. Zelcer N, Khanlou N, Clare R, Jiang Q, Reed-Geaghan EG, Landreth GE, Vinters HV, Tontonoz P (2007) Attenuation of neuroinflammation and Alzheimer's disease pathology by liver x receptors. Proc Natl Acad Sci U S A 104:10601-10606. https://doi.org/10.1073/pnas.0701096104

65. Ziegler-Waldkirch S, d'Errico P, Sauer JF, Erny D, Savanthrapadian S, Loreth D, Katzmarski N, Blank T, Bartos M, Prinz M et al (2018) Seed-induced Abeta deposition is modulated by microglia under environmental enrichment in a mouse model of Alzheimer's disease. EMBO J 37:167-182. https://doi.org/10. 15252/embj.201797021

66. Ziegler-Waldkirch S, Marksteiner K, Stoll J, d'Errico P, Friesen M, Eiler D, Neudel L, Sturn V, Opper I, Datta M et al (2018) Environmental enrichment reverses Abeta pathology during pregnancy in a mouse model of Alzheimer's disease. Acta Neuropathol Commun 6:44. https://doi.org/10.1186/s40478-018-0549-6

\section{Publisher's Note}

Springer Nature remains neutral with regard to jurisdictional claims in published maps and institutional affiliations.

Ready to submit your research? Choose BMC and benefit from:

- fast, convenient online submission

- thorough peer review by experienced researchers in your field

- rapid publication on acceptance

- support for research data, including large and complex data types

- gold Open Access which fosters wider collaboration and increased citations

- maximum visibility for your research: over $100 \mathrm{M}$ website views per year

At $\mathrm{BMC}$, research is always in progress.

Learn more biomedcentral.com/submission 